\title{
Phonon-Phonon Interactions in Strongly Bonded Solids: Selection Rules and Higher-Order Processes
}

\author{
Navaneetha K. Ravichandran* \\ Department of Mechanical Engineering, Indian Institute of Science, Bangalore 560012, India \\ David Broido \\ Department of Physics, Boston College, Chestnut Hill, Massachusetts 02467, USA
}

(Received 9 November 2019; revised manuscript received 5 March 2020; accepted 28 April 2020; published 18 June 2020)

\begin{abstract}
We show that the commonly used lowest-order theory of phonon-phonon interactions frequently fails to accurately describe the anharmonic phonon decay rates and thermal conductivity $(\kappa)$, even among strongly bonded crystals. Applying a first-principles theory that includes both the lowest-order three-phonon and the higher-order four-phonon processes to 17 zinc blende semiconductors, we find that the lowest-order theory drastically overestimates the measured $\kappa$ for many of these materials, while inclusion of four-phonon scattering gives significantly improved agreement with measurements. We identify new selection rules on three-phonon processes that help explain many of these failures in terms of anomalously weak anharmonic phonon decay rates predicted by the lowest-order theory competing with four-phonon processes. We also show that zinc blende compounds containing boron (B), carbon (C), or nitrogen (N) atoms have exceptionally weak four-phonon scattering, much weaker than in compounds that do not contain B, C, or N atoms. This new understanding helps explain the ultrahigh $\kappa$ in several technologically important materials like cubic boron arsenide, boron phosphide, and silicon carbide. At the same time, it not only makes the possibility of achieving high $\kappa$ in materials without $\mathrm{B}, \mathrm{C}$, or $\mathrm{N}$ atoms unlikely, but it also suggests that it may be necessary to include four-phonon processes in many future studies. Our work gives new insights into the nature of anharmonic processes in solids and demonstrates the broad importance of higher-order phonon-phonon interactions in assessing the thermal properties of materials.
\end{abstract}

DOI: 10.1103/PhysRevX.10.021063

\section{INTRODUCTION}

Phonon-phonon interactions arise from the inherent anharmonicity of the interatomic bonds in solids. They determine fundamental properties of crystals such as the lattice thermal conductivity $(\kappa)$ as well as the infrared, Raman, and neutron scattering cross sections [1-3]. They impact diverse phenomena including phonon drag [4], phonon bottleneck [5,6], and hydrodynamic thermal transport $[7,8]$.

In his pioneering work, Peierls described intrinsic thermal resistance in semiconductors and insulators using the lowest-order theory of phonon-phonon interactions, which involves three phonons [1]. The complexity of this

\footnotetext{
*navaneeth@iisc.ac.in
}

Published by the American Physical Society under the terms of the Creative Commons Attribution 4.0 International license. Further distribution of this work must maintain attribution to the author(s) and the published article's title, journal citation, and DOI.

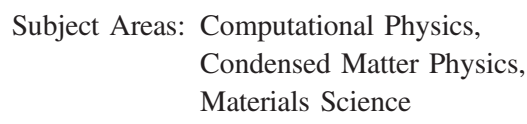

theory forced simple model approximations to be used until the past decade, when these simple models have been supplanted by first-principles computational approaches to describe anharmonic phonon decay and heat conduction [9-11], still based on the lowest-order theory.

While this lowest-order theory has been generally assumed to adequately describe anharmonic properties of crystals, $a b$ initio descriptions of higher-order four-phonon interactions are now becoming possible [12-21]. One might expect higher-order phonon-phonon interactions to be important in strongly anharmonic materials, i.e., those with weak chemical bonds. Indeed, recent work has shown that, for such materials, not only are four-phonon processes important [14-16], but the anharmonic renormalization of phonon modes themselves [14-16,22-30] is required to accurately match measured phonon frequency, thermal expansion, and thermal conductivity data.

But what about weakly anharmonic materials, i.e., those with relatively strong interatomic bonding? How well does the lowest-order theory work for such cases? Significant failures of the lowest-order theory have been found recently in cubic boron arsenide (BAs) $[13,17]$, boron antimonide 


\section{$300 \mathrm{~K}$}
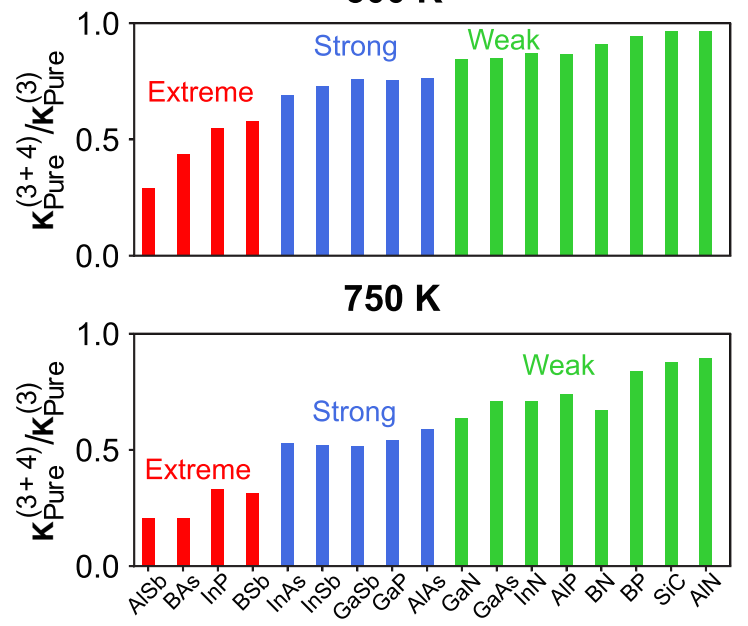

FIG. 1. Ratios of the thermal conductivities including fourphonon scattering to those omitting it $\left(\kappa_{\text {Pure }}^{(3+4)} / \kappa_{\text {Pure }}^{(3)}\right)$ for 17 zinc blende compounds at 300 (top) and $750 \mathrm{~K}$ (bottom). The large thermal conductivity reductions even at $300 \mathrm{~K}$ show that the lowest-order theory of phonon-phonon interactions is failing for many of these materials.

(BSb) [19], and aluminum antimonide (AlSb) [20]. But the underlying reasons for the failures in BAs and BSb apply to very few materials, and a full explanation for the failure in AlSb is not given in Ref. [20], as described below. At the same time, the lowest-order theory is found to work well for diamond [13,14], gallium nitride (GaN) [20], and cubic boron nitride $(\mathrm{BN})$ [18]. The computational cost of fourphonon calculations has thus far restricted consideration to only this small number of weakly anharmonic compounds. As a result, to date, a number of fundamental questions about the nature of anharmonic phonon-phonon processes in solids remain unanswered. Specifically, are the failures of the lowest-order theory of phonon-phonon interactions rare or common among weakly anharmonic materials? Is there a general framework to understand the interplay between three-phonon and four-phonon processes that can drive the failures? How do four-phonon scattering strengths vary across such materials? Can new trends be identified to aid in the search for materials with high $\kappa$ ?

To address these questions, in this work, we examine the anharmonic phonon decay rates and thermal conductivities of 17 different zinc blende (ZB) semiconductors with widely varying phonon properties using a first-principles computational approach that includes both the lowest-order three-phonon and higher-order four-phonon interactions as well as interactions between phonons and the isotopic mass disorder found in most crystals. The findings for $\kappa$ are summarized in Fig. 1 and confirmed by the available measured data for these materials (shown later). It is evident from the figure that failures of the lowest-order theory are remarkably common among ZB compounds, with nine of them showing a reduction in $\kappa$ of at least $20 \%$ at room temperature and at least $40 \%$ at $750 \mathrm{~K}$. For many of the 17 compounds, anharmonic decay rates of the optic phonons from four-phonon scattering are as large or even larger than those predicted by the lowest-order threephonon theory. Along with the previously identified compounds-BAs, BSb, and AlSb, we find a particularly catastrophic failure of the lowest-order theory to occur for indium phosphide (InP). Yet, the properties responsible for the failures in BAs and BSb cannot explain the behavior in InP, as we show below. The same is true of the significant failures seen in gallium antimonide (GaSb), indium arsenide (InAs), and indium antimonide ( $\mathrm{InSb}$ ). At the same time, the lowest-order theory works quite well for many compounds containing boron, carbon, or nitrogen atoms.

To fundamentally understand these findings, we first identify a set of selection rules that follow from energy and quasimomentum conservation on the lowest-order threephonon scattering and are dictated entirely by features in the phonon dispersions. To the extent that a material's phonon dispersions activate these selection rules, the phase space for the corresponding lowest-order three-phonon scattering channels is weakened or frozen out, which can result in unusually long lifetimes for both acoustic and optic phonons with correspondingly large contributions to $\kappa$, predicted in the lowest-order theory. While some of these selection rules have been identified previously [3133 ], they cannot explain the behavior found in many of the studied compounds. We identify new selection rules that are critical for developing a full understanding of the failures of the lowest-order theory in the zinc blende compounds.

Next, we show that, in striking contrast, selection rules do not affect the four-phonon scattering rates, which generally increase with frequency for all 17 materials. Thus, whenever the selection rules on the lowest-order processes drive weak three-phonon scattering, four-phonon scattering can become more important. Most remarkably, the materials naturally divide themselves into two groups according to the strength of the four-phonon scattering processes. In the materials not containing boron, carbon, or nitrogen (non-BCN compounds), the four-phonon scattering rates are of similar strength. For materials containing boron, carbon, or nitrogen (BCN compounds), four-phonon scattering rates are up to an order of magnitude smaller than those in the non-BCN compounds, a difference we trace to the unusually strong bonding in the $\mathrm{BCN}$ compounds. As expanded on below, the large difference in the strength of four-phonon scattering in the non-BCN compounds compared with the $\mathrm{BCN}$ compounds has profound implications on the phonon lifetimes and $\kappa$.

\section{SELECTION RULES ON PHONON-PHONON PROCESSES}

We begin by describing six selection rules for the lowestorder phonon-phonon scattering and discuss their interplay 
with the higher-order interactions. Some of these selection rules have been discussed previously [31-33]. We focus on crystals with two different atoms in the unit cell, which encompasses a large number of semiconductors and insulators including the technologically important zinc blende compounds studied in the present work, which have inherently weak anharmonicity of the crystal bonds. This fact is demonstrated by the small thermal expansion and weak effects of anharmonic renormalization on their phonon frequencies (see Supplemental Material [34], Sec. S1 for phonon dispersions and Sec. S2 for details on thermal expansion for all 17 compounds). At the same time, the breadth of the considered materials provides simple yet sufficiently different phonon dispersions that enable exploration of the effects of various selection rules, coexisting to different extents in different materials, on their anharmonic phonon decay rates and thermal conductivities.

\section{A. Lowest-order processes}

We divide the six phonon polarizations (branches) into three acoustic $(A)$ and three optic $(O)$ branches. There are four possible combinations of the lowest-order anharmonic processes that involve the decay of a phonon into two others or the coalescence of two phonons into a third. These are (i) $(A A A)$; (ii) $(A A O)$; (iii) $(A O O)$; and (iv) $(O O O)$. These processes must satisfy conservation of the phonon energy and quasimomentum [35]:

$$
\begin{gathered}
\omega_{j}(\mathbf{q}) \pm \omega_{j^{\prime}}\left(\mathbf{q}^{\prime}\right)=\omega_{j^{\prime \prime}}\left(\mathbf{q}^{\prime \prime}+\mathbf{G}\right), \\
\mathbf{q} \pm \mathbf{q}^{\prime}=\mathbf{q}^{\prime \prime}+\mathbf{G} .
\end{gathered}
$$

Here, the three phonons participating in the scattering process have wave vectors $\mathbf{q}, \mathbf{q}^{\prime}$, and $\mathbf{q}^{\prime \prime}$, branches $j, j^{\prime}$, and $j^{\prime \prime}$, and frequencies $\omega_{j}(\mathbf{q}), \omega_{j^{\prime}}\left(\mathbf{q}^{\prime}\right)$, and $\omega_{j^{\prime \prime}}\left(\mathbf{q}^{\prime \prime}\right)$, respectively, and $\mathbf{G}$ is a reciprocal lattice vector to account for normal $(\mathbf{G}=0)$ and umklapp $(\mathbf{G} \neq 0)$ processes.

For each phonon mode, $(j, \mathbf{q})$, and fixed $j^{\prime}$ and $j^{\prime \prime}$, the phase space for the lowest-order scattering is defined by the set of wave vectors $\mathbf{q}^{\prime}$ and $\mathbf{q}^{\prime \prime}$ that satisfy Eqs. (1) and (2) $[9,10,36-38]$. Features in the phonon dispersions that cause the phase space to vanish or significantly weaken define selection rules on these processes.

First, we note a selection rule on $O O O$ processes: Energy conservation freezes out $O O O$ processes if the optic phonon bandwidth, $\Delta \omega_{O}$, is smaller than the lowest optic phonon frequency. This selection rule is satisfied in all compounds studied in the present work, so we do not discuss the $O O O$ selection rule further. We identify five selection rules on $A A A, A A O$, and $A O O$ processes that follow directly from Eqs. (1) and (2), combining those previously found [31-33] with two new ones. These selection rules divide into two types that complement each other. The first two selection rules (classified as type 1 selection rules) follow directly from energy conservation [Eq. (1)].

\section{Type 1 selection rules}

(1) AOO\#1 selection rule.-AOO processes cannot occur involving acoustic phonons whose frequencies exceed the optic phonon bandwidth. This selection rule establishes a cutoff frequency $\left(\Delta \omega_{O}\right)$ in the acoustic phonon spectrum beyond which an acoustic phonon cannot participate in $A O O$ processes. This cutoff has been pointed out previously [33].

(ii) AAO selection rule.-An $A A O$ process cannot occur if one of the participating acoustic phonons has a frequency smaller than the frequency gap between acoustic and optic phonons. This selection rule establishes a cutoff frequency for the acoustic phonons, $\Delta \omega_{A-O}$ - the $A-O$ frequency gap, below which an acoustic phonon cannot participate in $A A O$ processes. It also establishes a cutoff frequency for the optic phonons, $2 \Delta \omega_{A}\left(\Delta \omega_{A}\right.$ is the acoustic phonon bandwidth), above which optic phonons cannot participate in $A A O$ processes. From this selection rule, it follows that, for materials with $A-O$ gaps larger than the highest acoustic phonon frequency $\Delta \omega_{A}, A A O$ processes are completely forbidden [32].

If $\Delta \omega_{O}<\Delta \omega_{A-O}$, the $A A O$ and $A O O \# 1$ selection rules act in unison to create a frequency window for the acoustic phonons where both $A A O$ and $A O O$ scattering channels are completely forbidden and only the $A A A$ three-phonon scattering channel is allowed. This case is illustrated schematically in Figs. 2(b) and 2(d). Similarly, if $\Delta \omega_{O}>$ $\Delta \omega_{A}-\Delta \omega_{A-O}$, then a frequency window is created where only $A O O$ scattering by optic phonons can occur. This case is illustrated in Fig. 2(e). If frequency windows are created by the $A A O$ and $A O O \# 1$ selection rules, then the following three selection rules (classified as type 2 selection rules) can drive anomalously weak three-phonon scattering.

\section{Type 2 selection rules}

(3) AAA\#1 selection rule.-An anharmonic process of any order in which one phonon decays into a set of other phonons with higher phase velocity cannot occur. This selection rule was proven by Lax, Hu, and Narayanamurti [31].

(4) AAA\#2 selection rule.-As the group velocities of longitudinal acoustic (LA) phonons increases compared with transverse acoustic (TA) phonon velocities, the phase space of low-frequency $A A A$ processes decreases. To our knowledge, this selection rule has not been pointed out before. Unlike the other selection rules, this one does not completely 
(a)

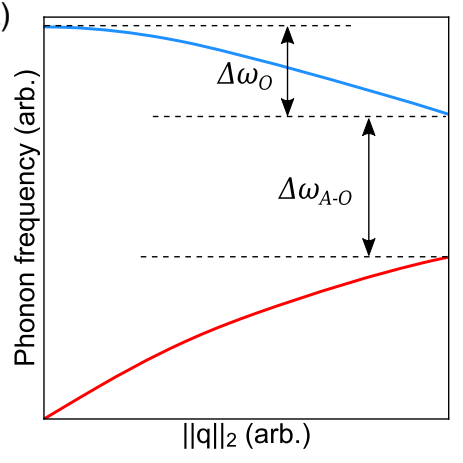

(b)

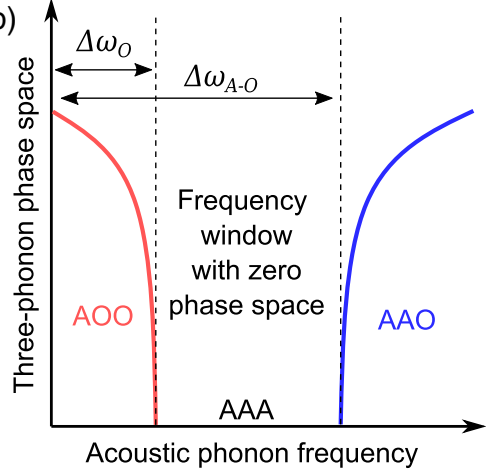

(c)

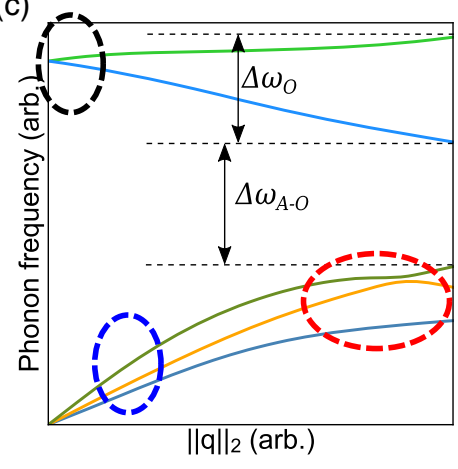

(d)

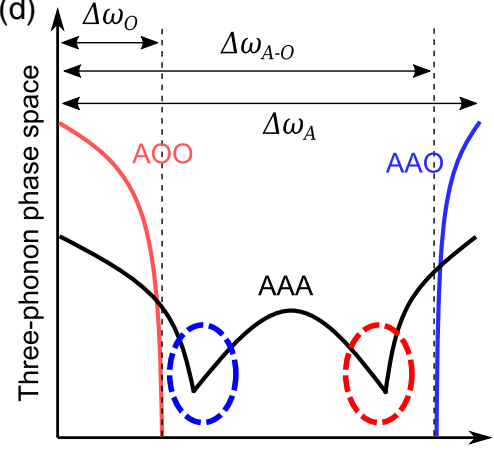

Acoustic phonon frequency (e)

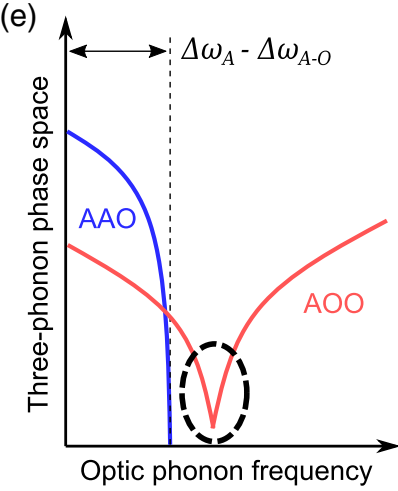

FIG. 2. Hypothetical phonon dispersions (a) and the corresponding three-phonon phase space (b), where degenerate acoustic phonons, a small optic phonon bandwidth $\left(\Delta \omega_{O}\right)$, and a large $A-O$ frequency gap $\left(\Delta \omega_{A-O}\right)$ result in a frequency window for the acoustic phonons without any phase space for three-phonon scattering (due to $A A O, A O O \# 1$ and $A A A \# 1$ selection rules). Also shown are the features of the dispersions of a real material (c), where acoustic phonons having a large LA-TA velocity ratio (blue dashed oval) and/or being bunched together (red dashed oval) along with small $\Delta \omega_{O}$ and a large $A-O$ gap result in a frequency window with weak total three-phonon scattering phase space due to $A A O, A O O \# 1, A A A \# 1$, and $A A A \# 2$ selection rules (d). Furthermore, near degeneracy in the optic phonons [black dashed oval in (c) and (e)] along with a large $A-O$ gap can result in weak three-phonon scattering phase space for the optic phonons due to $A A O$ and $A O O \# 2$ selection rules. The curves labeled $A A A, A A O$, and $A O O$ in (b), (d), and (e) show schematically the regions in which the corresponding three-phonon processes occur.

forbid any particular three-phonon scattering channel except in the limit of infinite LA/TA phonon group velocity ratio. However, the trend it expresses is critical in explaining the anomalously small acoustic three-phonon scattering rates in several zinc blende compounds discussed in the next section. This selection rule is examined in more detail in Supplemental Material, Sec. S8(B) [34].

(5) AOO\#2 selection rule.-An $A O O$ process is forbidden when both optic phonons derive from the same phonon branch, provided the group velocities of the optic phonons are smaller than those of the acoustic phonons. To our knowledge, this selection rule has not been pointed out before. Typically, the group velocities of optic phonons are smaller than those of the acoustic phonons that can participate in an $A O O$ process while satisfying energy conservation. We have confirmed numerically that this selection rule is satisfied for all 17 materials in this study. Exceptions can occur in materials with larger optic phonon group velocities, such as the lead chalcogenides.
Further discussion is given in Supplemental Material, Sec. S8(A) [34].

\section{B. Minimal influence of selection rules on four-phonon processes}

Ziman suggested that selection rules on four-phonon processes are less restrictive than those on three-phonon processes [35]. Selection rules do exist for some fourphonon processes. For example, the general selection rule noted in Ref. [31] forbids $A \leftrightarrow A+A+A$ processes when all phonons derive from the same branch. However, it does not forbid the corresponding $A+A \leftrightarrow A+A$ processes. Similarly, processes such as $A \leftrightarrow A+O+O$ do not conserve energy, while a large phase space exists for $A+$ $O \leftrightarrow A+O(A O A O)$ processes. Our quantitative firstprinciples calculations show that the $A O A O$ processes dominate in all 17 compounds studied in this work, with $A A A A$ and $O O O O$ processes also being important for acoustic and optic phonons, respectively, and the influence of selection rules on four-phonon processes is minimal. 


\section{Interplay of lowest-order selection rules and four-phonon scattering}

The above selection rules impose strict constraints on the phase space for specific three-phonon processes, which are governed completely by a material's phonon dispersionsa harmonic quantity. As described earlier, the selection rules complement each other: For materials whose constituent atoms have different masses (so as to create nonzero $\Delta \omega_{A-O}$ ) and relatively low ionicity (so that $\Delta \omega_{O}$ is relatively small), $A A O$ and $A O O \# 1$ selection rules create frequency windows where only $A A A$ scattering can occur for acoustic phonons and only $A O O$ scattering can occur for optic phonons. Then, if features in the material's phonon dispersions activate $A A A \# 1, A A A \# 2$, or $A O O \# 2$ selection rules, regions of small total three-phonon phase space result. In principle, if hypothetical phonon dispersions existed with a collection of features described in the above selection rules, then the lowest-order theory would predict infinite intrinsic lifetimes and dissipationless transport for the affected phonon modes. For example, if the three acoustic phonon branches of hypothetical phonon dispersions coincided throughout the Brillouin zone (BZ), then the $A A A \# 1$ selection rule would require that the phase space for $A A A$ scattering vanish. Then, if $\Delta \omega_{A-O}>\Delta \omega_{O}$, a frequency window would be created with zero phase space for lowest-order phonon-phonon scattering for acoustic phonons, according to $A O O \# 1$ and $A A A \# 1$ selection rules. This scenario is illustrated in Figs. 2(a) and 2(b). Similarly, if all of the optic phonon branches of hypothetical phonon dispersions coincided throughout the BZ, the optic phonon velocities were much smaller than those of the acoustic phonons available that satisfy energy conservation, and $\Delta \omega_{A-O}>\Delta \omega_{A}$, then the optic phonons could not decay at all due to $A A O$ and $A O O \# 2$ selection rules (no separate plot is shown for the optic phonon phase space, since it would be uniformly zero in this scenario). Although these scenarios do not occur in real materials, insofar as features in the real dispersions approach those in the hypothetical ones, selection rules are activated that reduce the phase space for the lowest-order processes. For example, if acoustic branches have similar frequencies in some region of the $\mathrm{BZ}$, then the phase space for some $A A A$ processes involving phonons with frequencies in this region becomes small. If $A A O$ and $A O O \# 1$ selection rules create a frequency window where the $A A A$ processes are weak, the total three-phonon scattering phase space will be small, as shown in Fig. 2(d). This behavior is found in BAs and $\mathrm{BSb}$, as explained previously [32]. Similarly, if phonons from different optic branches become nearly degenerate in some region of the $\mathrm{BZ}$ and their frequencies are larger than $\Delta \omega_{A}-\Delta \omega_{A-O}$, then the phase space for the corresponding $A O O$ processes becomes weak. This scenario is illustrated in Fig. 2(e). We show in the next section that it explains the large contributions to the three-phonon limited thermal conductivity from optic phonons in BAs and AlSb. We note that the degree of activation of the type 2 selection rules is qualitative and cannot be fully assessed by visual inspection of phonon dispersions. Quantitative ab initio calculations are required to determine the impact of the selection rules on the anharmonic decay rates and thermal conductivity of a given material.

Higher-order four-phonon scattering rates are generally weaker than their lowest-order counterparts in weakly anharmonic materials at low-to-moderate temperatures. However, here, we emphasize that, when features in the phonon dispersions cause the above-described selection rules to impose severe constraints on the phase space for the lowest-order phonon-phonon processes, the effects of fourphonon scattering can become quite important. In the next section and in Supplemental Material, Sec. S3 [34], the calculated thermal conductivities of the studied 17 different materials are presented and compared to measured data, and the failures and successes of the lowest-order theory are explained in terms of the selection rules and their interplay with the varying strengths of four-phonon scattering.

\section{RESULTS}

The thermal conductivities of the 17 compounds with zinc blende structure- $\mathrm{GaAs}, \mathrm{AlP}, \mathrm{AlAs}, \mathrm{GaP}, \mathrm{GaSb}$, InAs, $\mathrm{InSb}, \mathrm{AlSb}, \mathrm{InP}, \mathrm{BN}, \mathrm{BP}, \mathrm{BAs}, \mathrm{BSb}, \mathrm{SiC}, \mathrm{GaN}, \mathrm{InN}$, and AlN-are obtained as functions of the temperature by solving the Boltzmann equation for phonon transport including three-phonon, four-phonon, and phonon-isotope scattering using a recently developed first-principles approach [14]. A brief description of this approach is included in Sec. VII and the Appendix. Of the 17 materials considered here, all but five (AlP, GaN, InN, AlN, and BSb) have measured thermal conductivity data available for comparison. Six such comparisons are presented in Fig. 3 for BAs, AlSb, and InP and Fig. 4 for GaSb, InAs, and InSb, while the remaining (except $\mathrm{BN}$ ) are given in Supplemental Material, Sec. S3 [34]. The case of BN is extensively described in the main text and the supplemental information of our recent publication [18], so we do not present those plots in Supplemental Material [34]. Calculated thermal conductivities are given for the following four conditions: lowest-order three-phonon scattering only $\left(\kappa_{\text {Pure }}^{(3)}\right)$, three-phonon scattering and phonon scattering from the natural isotopic disorder $\left(\kappa_{\text {Nat }}^{(3)}\right)$, three-phonon and four-phonon scattering only $\left(\kappa_{\text {Pure }}^{(3+4)}\right)$, and three-phonon, four-phonon, and phonon-isotope scattering $\left(\kappa_{\mathrm{Nat}}^{(3+4)}\right)$. Calculated room temperature (RT) $\kappa$ values and convergence studies for each material are tabulated in Supplemental Material, Tables SII and SIII [34]. In many of the cases, $\kappa(T)$ calculated from the lowest-order theory significantly overestimates the measured $\kappa(T)$, while including fourphonon interactions brings the calculated $\kappa(T)$ curves into very good agreement with the measured data $[17,18,39$ 50]. As already seen in Fig. 1, four-phonon scattering 
(a)

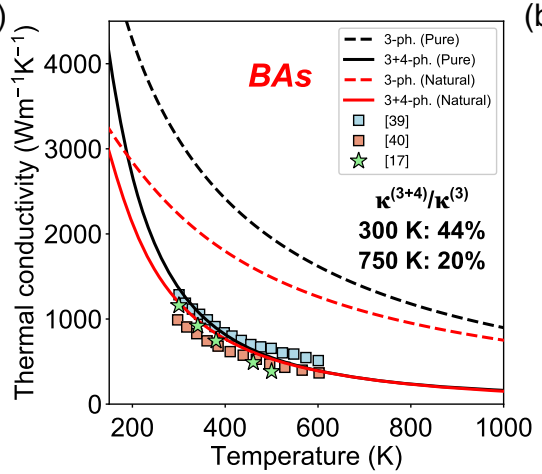

(b)

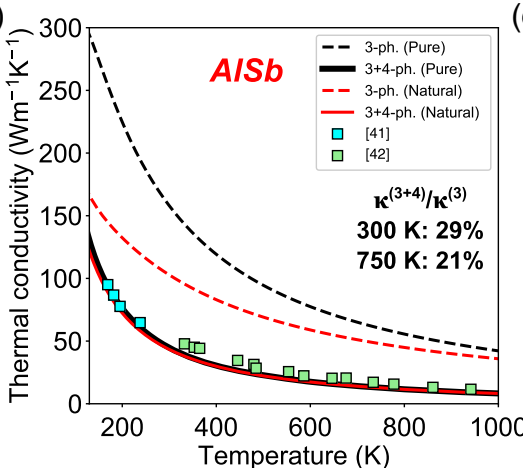

(c)

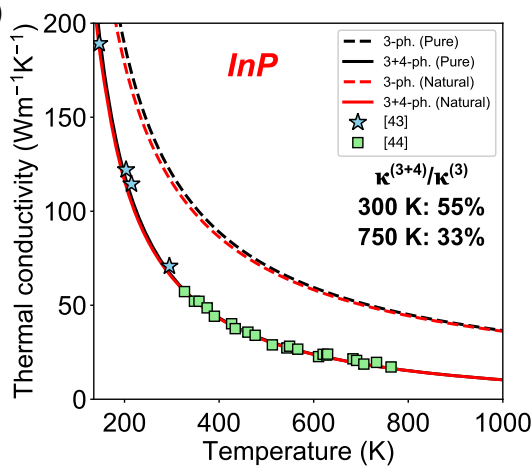

FIG. 3. First-principles computed $\kappa_{\text {Pure }}^{(3)}$ (dashed black line), $\kappa_{\text {Pure }}^{(3+4)}$ (solid black line), $\kappa_{\text {Nat }}^{(3)}$ (dashed red line), and $\kappa_{\text {Nat }}^{(3+4)}$ (solid red line) for BAs (a), AlSb (b), and InP (c) as functions of the temperature and compared with the experimental data in the literature. The experimental measurements of $\kappa$ are from (a) BAs, Kang et al. [39], Li et al. [40], and Tian et al. [17]; (b) AlSb, Muzhdaba et al. [41] and Steigmeier and Kudman [42]; and (c) InP, Aliev, Nashelskii, and Shalyt [43] and Kudman and Steigmeier [44].

(a)

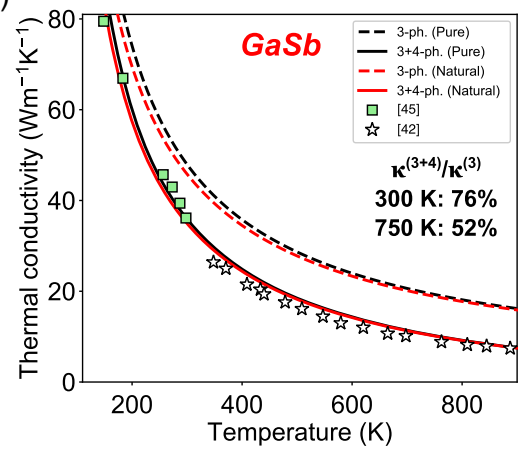

(b)

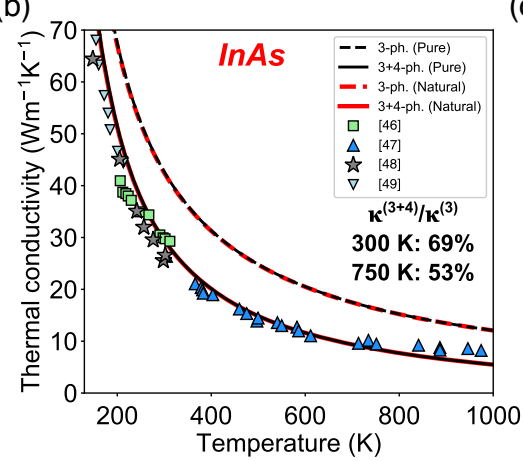

(c)

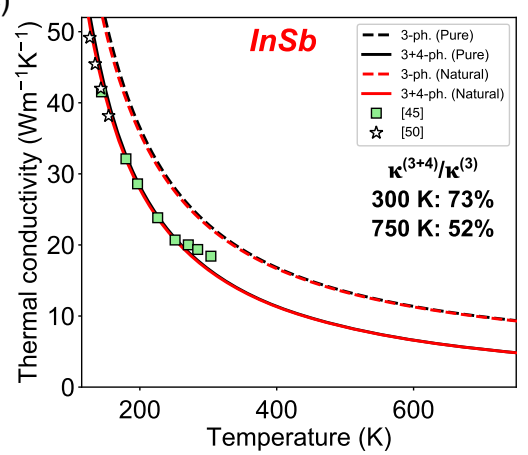

FIG. 4. First-principles computed $\kappa_{\text {Pure }}^{(3)}$ (dashed black line), $\kappa_{\text {Pure }}^{(3+4)}$ (solid black line), $\kappa_{\text {Nat }}^{(3)}$ (dashed red line), and $\kappa_{\text {Nat }}^{(3+4)}$ (solid red line) for $\mathrm{GaSb}(\mathrm{a})$, InAs (b), and InSb (c) as functions of the temperature and compared with the experimental data in the literature. The experimental measurements of $\kappa$ are from (a) GaSb, Holland [45] and Steigmeier and Kudman [42]; (b) InAs, Arasly, Ragimov, and Aliev [46], Bowers, Bauerle, and Cornish [47], Le Guillou and Albany [48], and Tamarin and Shalyt [49]; and (c) InSb, Holland [45] and Shalyt, Tamarin, and Ivleva [50].

suppresses the $\kappa_{\text {Pure }}^{(3)}$ for nine of the 17 compounds by at least $20 \%$ at RT $(300 \mathrm{~K})$ and at least $40 \%$ at $750 \mathrm{~K}$. The larger suppressions at $750 \mathrm{~K}$ are explained by the more rapid increase of four-phonon scattering rates with the temperature compared with three-phonon scattering rates $[13,19]$. Thus, it is clear that the lowest-order theory is failing for many of these compounds.

As explained in the previous section, activation of the type 1 selection rules can create frequency windows where only $A A A$ can occur for acoustic phonons and only $A O O$ processes can occur for optic phonons. Pockets of small three-phonon scattering phase space result if type 2 selection rules are simultaneously activated. The most significant effects from this combined activation of type 1 and type 2 selection rules occurs in four compoundsBAs, BSb, AlSb, and InP, those falling in the "extreme" classification in Fig. 1. Since different type 2 selection rules are required to explain the extreme behavior in these materials, in the results discussed below, materials are grouped according to which of the type 2 selection rules (AAA\#1, $A A A \# 2$, and $A O O \# 2)$ are activated. We note that the degree of activation of type 2 selection rules is somewhat qualitative but is evident from the three-phonon phase space plots.

\section{A. Materials influenced by $A A A \# 1$ selection rule: BAs, BSb, GaN, BP, BN, SiC, and AIN}

The seven compounds BAs, BSb, BP, BN, SiC, GaN, and AlN have acoustic branches that become bunched together, thereby activating the $A A A \# 1$ selection rule. The signature of this activation is the sharp dip in the $A A A$ scattering phase space [see Figs. 5(a), S6, S7(a), and S8(a) [34]]. BAs and BSb have been discussed in depth in the context of ultrahigh $\kappa$, where the $A A A \# 1, A A O$, and $A O O \# 1$ selection rules conspire to make three-phonon scattering unusually weak [32]. The large pnictide-to-B mass ratios of BAs and BSb (approximately 7 in BAs and approximately $11 \mathrm{in} \mathrm{BSb}$ ) give large $A-O$ gaps that 

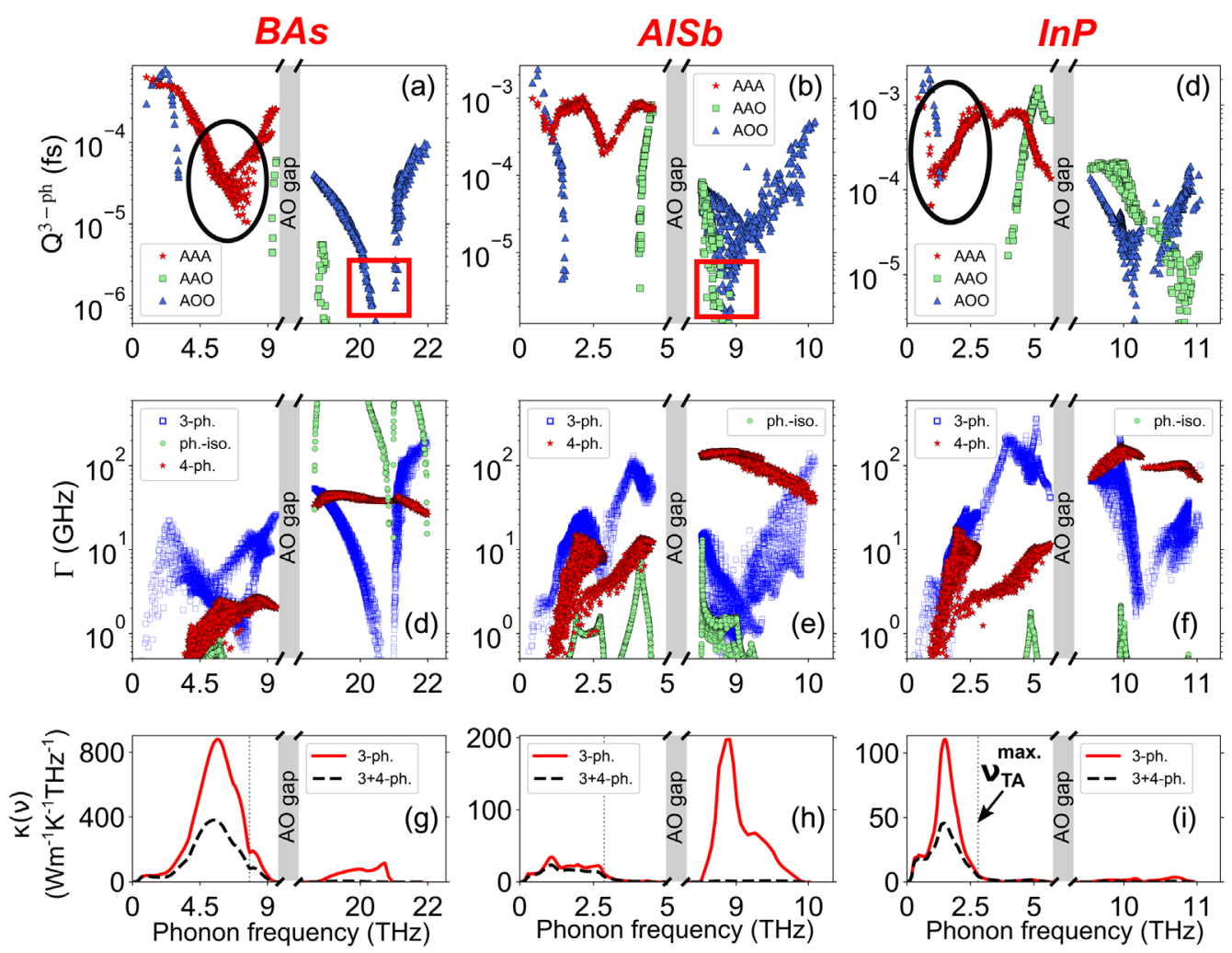

FIG. 5. Calculated three-phonon scattering phase space for different scattering channels (a)-(c), three-phonon, four-phonon, and phonon-isotope scattering rates at $300 \mathrm{~K}(\mathrm{~d})-(\mathrm{f})$, and spectral contributions to $\kappa_{\text {Pure }}^{(3)}$ (solid red line) and $\kappa_{\text {Pure }}^{(3+4)}$ (dashed black line) at $300 \mathrm{~K}$ (g)-(i), for BAs [first column, (a), (d), and (g)], AlSb [second column, (b), (e), and (h)], and InP [third column, (c), (f), and (i)]. The dotted vertical lines in (g)-(i) show the location of the maximum TA phonon frequency $\left[\nu_{\mathrm{TA}}^{\max }=\omega_{\mathrm{TA}}^{\max } /(2 \pi)\right]$ in each material. The black oval regions in (a) and (c) indicate the weak $A A A$ phase space due to $A A A \# 1$ (BAs) and $A A A \# 2$ (InP) selection rules in the frequency windows opened up by vanishing $A A O$ and $A O O$ scattering rates from the $A A O$ and $A O O \# 1$ selection rules in both materials. The red square regions in (a) and (b) show the weak $A O O$ scattering rates from the $A O O \# 2$ selection rule in BAs and AlSb, respectively. The spectral $\kappa_{\text {Pure }}^{(3)}$ for AlSb (h) also shows an anomalously large contribution from the optic phonons, which is completely suppressed by four-phonon scattering.

completely freeze out $A A O$ scattering, and the narrow optic phonon bandwidths constrain $A O O$ scattering of the acoustic phonons to low frequencies [see Figs. S1(c) and S1(d) [34] for phonon dispersions and Figs. 5(a) and S6(c) for the three-phonon phase space for BAs and BSb, respectively]. As seen in these figures, these two selection rules expose a large frequency window of only $A A A$ scattering processes. This window combined with the activation of the $A A A \# 1$ selection rule gives a small phase space for the lowest-order processes and results in significantly weaker three-phonon scattering rates and large contributions to $\kappa_{\text {Pure }}^{(3)}$ [see Figs. 5(a), 5(d), and 5(g) for the results for BAs and Figs. S6(c), S13(c), and S16(c) [34] for those of $\mathrm{BSb}]$. Upon including the four-phonon scattering, the RT $\kappa_{\text {Pure }}^{(3)}$ of BAs is sharply reduced from 3100 to around $1400 \mathrm{Wm}^{-1} \mathrm{~K}^{-1}$. In spite of this large reduction, we show below that the four-phonon scattering in BAs is, in fact, much weaker for the acoustic phonons in the frequency range where the $A A A$ three-phonon scattering rates are small than in many other materials. We note that our calculations of $\kappa$ including three-phonon, fourphonon, and phonon-isotope scattering are in good agreement with the measured data $[17,39,40]$ [Fig. 3(a)].

The much smaller heavy-to-light atom mass ratios in $\mathrm{BN}$, $\mathrm{SiC}, \mathrm{BP}$, and AlN result in smaller $A-O$ gaps in these materials. As a result, $A A O$ scattering dominates in the same frequency region as the dips in the $A A A$ scattering rates. The implications of this masking of weak $A A A$ scattering are discussed below. The large $\kappa_{\text {Pure }}^{(3)}$ in these materials occurs for the conventional reasons: light atoms and stiff chemical bonds. Measured values [18,51-54] are close to those predicted by the lowest-order theory. GaN has a relatively large Ga-to-N mass ratio of 5, which gives a large enough $A-O$ gap to partially expose the dip in the $A A A$ scattering rates [Fig. S8(a) [34]]. This contributes to a relatively large $\mathrm{RT} \kappa_{\text {Pure }}^{(3)} \sim 400 \mathrm{Wm}^{-1} \mathrm{~K}^{-1}$, consistent with prior calculations 55]], but far lower than the RT $\kappa_{\text {Pure }}^{(3)} \sim 3000 \mathrm{Wm}^{-1} \mathrm{~K}^{-1}$ obtained for BAs.

In $\mathrm{BP}, \mathrm{BN}, \mathrm{SiC}, \mathrm{GaN}, \mathrm{InN}$, and $\mathrm{AlN}$, inclusion of fourphonon scattering has only a weak effect on $\kappa(3 \%, 5 \%, 9 \%$, 
$4 \%, 13 \%$, and $15 \%$ reductions in $\kappa_{\text {Pure }}^{(3)}$ at $\mathrm{RT}$ for $\mathrm{SiC}, \mathrm{BP}$, $\mathrm{BN}, \mathrm{AlN}, \mathrm{InN}$, and GaN, respectively) as seen in Fig. S5 [34] and in Ref. [18]. This effect is explained in part by the remarkably weak four-phonon scattering rates, which are found to be characteristic of the compounds with B, C, and $\mathrm{N}$ atoms, as discussed in detail below.

\section{B. Materials influenced by $A A A \# 2$ selection rule: InP, InAs, InSb, and GaSb}

Figure 6 ranks the ratio of LA to TA phonon group velocities, averaged over the center quarter of the BZ around the $\Gamma$ point for all 17 compounds considered in this work. Note that the choice of $1 / 4$ of the BZ is somewhat arbitrary, and the same ordering is obtained by choosing, e.g., 1/5 of the BZ. The four compounds InP, InAs, InSb, and $\mathrm{GaSb}$ show the largest differences between the averaged LA and TA phonon group velocities and so more strongly activate the AAA\#2 selection rule. The most pronounced effect occurs in InP. The large In-to-P mass ratio of 3.7 in InP creates a large $A-O$ gap, and the optic phonon bandwidth is small [see Fig. S4(b) [34]]. Thus, $A A O$ and $A O O \# 1$ selection rules create a frequency window (1.5-3.5 THz) where only $A A A$ scattering processes occur [red stars in Fig. 5(c)]. There, a significantly reduced phase space for $A A A$ scattering results in the lowfrequency region [black oval in Fig. 5(c)]. The striking effects of this restriction are seen in the spectral contributions to $\kappa_{\text {Pure }}^{(3)}$ at RT shown in Fig. 5(i), where a large peak occurs around $1.5-2 \mathrm{THz}$.

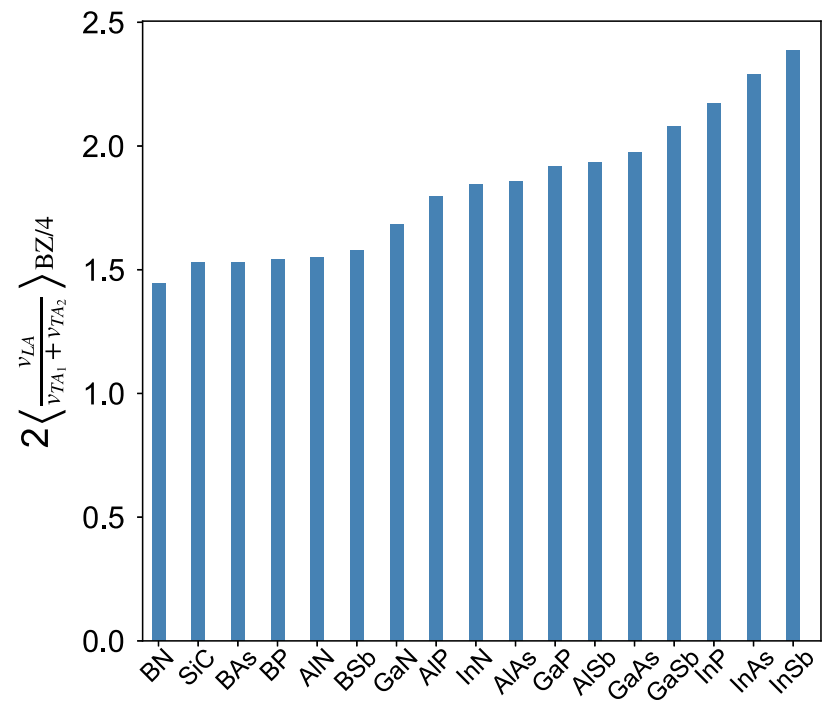

FIG. 6. Ratios of the LA phonon velocities to the mean TA phonon velocities averaged over $1 / 4$ of the BZ centered at the $\Gamma$ point for 17 zinc blende compounds. The compounds are listed in ascending order of the mean LA-TA velocity ratio. Compounds with the largest velocity ratios have the $A A A \# 2$ selection rule activated.
In the 1.5-2 THz frequency region of weak $A A A$ scattering, the RT four-phonon scattering rates are found to be comparable to or even exceed those of the three-phonon processes [Fig. 5(f)], which results in a large suppression by around $45 \%$ at $300 \mathrm{~K}$, which increases to almost $70 \%$ at $750 \mathrm{~K}$. These remarkably large reductions are supported by the measured data for InP [43,44] as shown in Fig. 3(c), which is in excellent agreement with the results including four-phonon scattering over a wide temperature range.

Large differences in the LA and TA velocities in InAs, $\mathrm{InSb}$, and $\mathrm{GaSb}$ (Fig. 6) also activate the $A A A \# 2$ selection rule, which gives the low-frequency dips in the $A A A$ phase space identified by the black ovals in Figs. 7(a)-7(c). However, the smaller heavy-to-light atom mass ratios compared to InP result in smaller frequency windows, where the dips in the $A A A$ phase space occur are exposed. Since the dominant contributions to $\kappa$ from the lowest-order theory occur in this frequency region, where four-phonon scattering is also strong (see Fig. 7), a significant suppression in $\kappa_{\text {Pure }}^{(3)}$ results. Four-phonon scattering suppresses the $\kappa_{\text {Pure }}^{(3)}$ for each compound in this group, ranging from around $25 \%-30 \%$ at RT up to around $40 \%-50 \%$ at $750 \mathrm{~K}$.

\section{Materials influenced by $A O O \# 2$ selection rule: BAs, AlSb, AlAs, AlP, BP, GaAs, GaSb, GaP, InAs, and InSb}

Optic phonon modes have only two possible channels for the lowest-order anharmonic decay: $A A O$ and $A O O$. The optic phonons in the ten listed compounds have regions of near degeneracy and so are strongly influenced by the $A O O \# 2$ selection rule, as seen from the sharp dips in the phase space for $A O O$ scattering [see Figs. 5(a) and 5(b), 7(a)-7(c), S6(b), S7(b) and S7(c), and S8(b) and S8(c) [34]]. However, the relatively small heavy-to-light atom mass ratios in AlAs, AlP, BP, GaAs, GaSb, GaP, InAs, and InSb give no frequency window to expose the weak $A O O$ scattering rates. Thus, the effects of the $A O O \# 2$ selection rule on optic phonon lifetimes are masked in these compounds. The most extreme effects occur in BAs and AlSb, because the large $A-O$ gaps create frequency windows where $A A O$ scattering is frozen out, thus exposing the regions of weak $A O O$ scattering. The unusually weak ionicity in BAs [56] results in a near degeneracy of the three optic branches near the $\Gamma$ point of the BZ [see Supplemental Material, Fig. S1(c) [34] ]. Then, the actions of $A A O$ and $A O O \# 2$ selection rules result in an exceptionally small phase space for $A O O$ scattering for the longitudinal optic (LO) and transverse optic (TO) phonons at the $\Gamma$ point [Fig. 5(a), red box]. As a result, large room temperature contributions to $\kappa$ of around $130 \mathrm{Wm}^{-1} \mathrm{~K}^{-1}$ are predicted from the lowest-order theory [Fig. 5(g)], significantly exceeding the total $\kappa$ of many common semiconductors. In contrast, $\mathrm{BSb}$ has a larger separation between the LO and TO phonons throughout the BZ [e.g., see Supplemental Material, Fig. S1(d) [34]]; thus, the AOO\#2 selection rule 

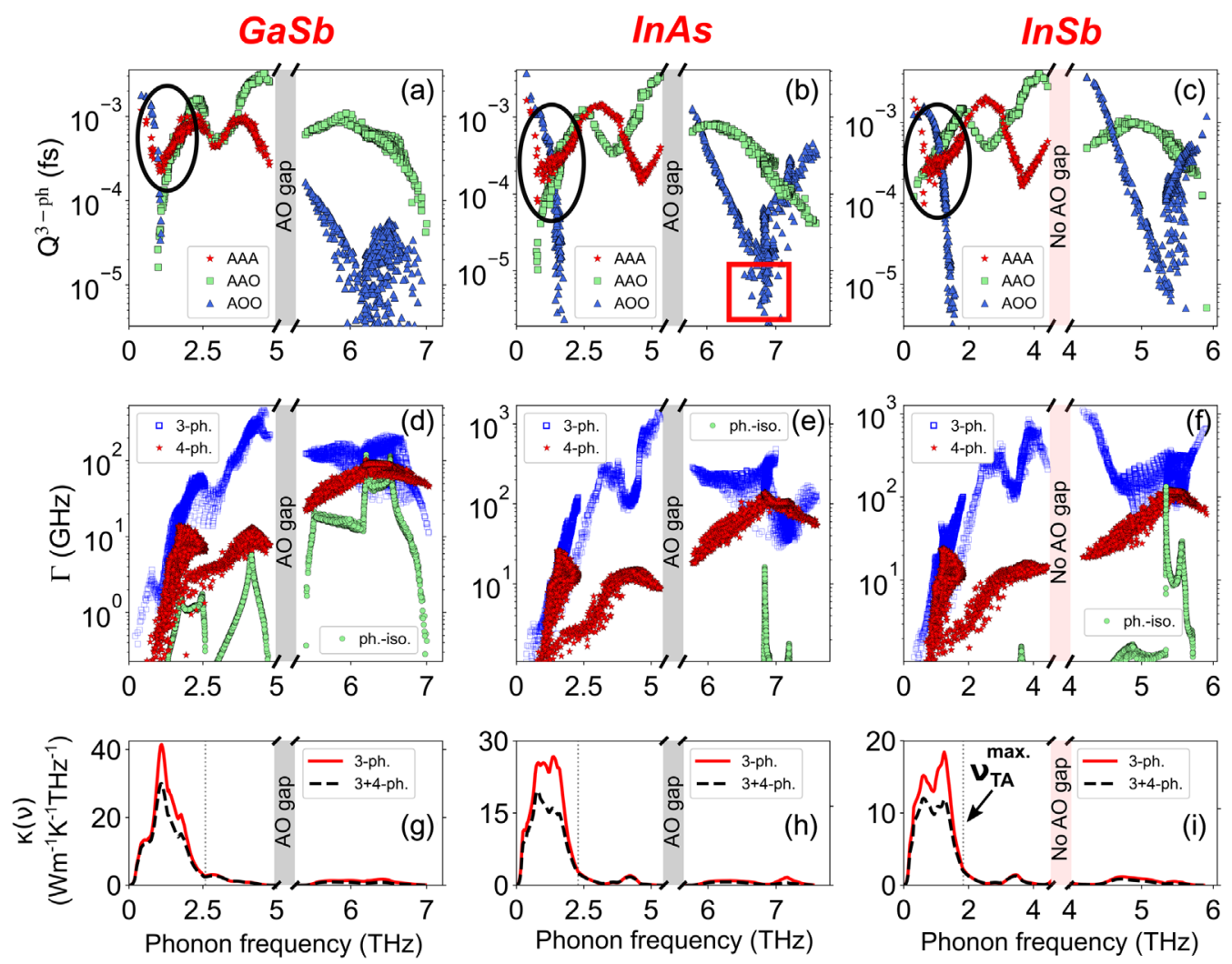

FIG. 7. Calculated three-phonon scattering phase space for different scattering channels (a)-(c), three-phonon, four-phonon, and phonon-isotope scattering rates at $300 \mathrm{~K}$ (d)-(f), and spectral contributions to $\kappa_{\text {Pure }}^{(3)}$ (solid red line) and $\kappa_{\text {Pure }}^{(3+4)}$ (dashed black line) at $300 \mathrm{~K}$ (g)-(i), for GaSb [first column, (a), (d), and (g)], InAs [second column, (b), (e), and (h)], and InSb [third column, (c), (f), and (i)]. The dotted vertical lines in (g)-(i) show the location of the maximum TA phonon frequency $\left[\nu_{\mathrm{TA}}^{\max }=\omega_{\mathrm{TA}}^{\max } /(2 \pi)\right]$ in each material. The black oval regions in (a)-(c) indicate weak $A A A$ phase space due to the $A A A \# 2$ selection rule. However, unlike in Figs. 5(a)-5(c), relatively strong $A A O$ and $A O O$ scattering channels partially overlap with the weak $A A A$ scattering frequency range, thus increasing the total three-phonon scattering rates in (d)-(f). The red square region in (b) shows the weak $A O O$ scattering rates from the $A O O \# 2$ selection rule, where, once again, the $A A O$ scattering channel is strong due to the small $A-O$ gap in InAs. Analogous behavior occurs in AlAs, AlP, BP, GaAs, GaSb, GaP, and InSb.

has little influence on the optic phonons, which contribute negligibly to $\kappa$.

As in BAs, the large $A-O$ gap in AlSb [Fig. S2(d) [34] ] resulting from the large $\mathrm{Sb}$-to-Al mass ratio creates a frequency window with no $A A O$ scattering and exposes the weak $A O O$ scattering phase space from the $A O O \# 2$ selection rule [Fig. 5(b), red box]. However, in stark contrast to BAs, this window occurs near the degeneracy of $\mathrm{LO}$ and $\mathrm{TO}_{2}$ optic phonon modes in regions of the $\mathrm{BZ}$ far from the zone center and away from high-symmetry directions [see Supplemental Material, Fig. S10(d) [34] ]. In these regions, $\mathrm{TO}_{2}+A \leftrightarrow \mathrm{LO}$ processes become vanishingly small, while $\mathrm{LO} \leftrightarrow \mathrm{TO}_{1}+A$ and $\mathrm{TO}_{2} \leftrightarrow \mathrm{TO}_{1}+$ $A$ scattering is weak. The resulting small lowest-order optic phonon scattering rates [Fig. 5(e)] give anomalously large contributions to $\kappa_{\text {Pure }}^{(3)}$ of around $90 \mathrm{Wm}^{-1} \mathrm{~K}^{-1}$ [Fig. 5(h)].

Four-phonon scattering in BAs and AlSb completely suppresses the optic phonon lifetimes [Figs. 5(d), 5(e), 5(g), and 5(h)], in striking contrast to predictions from the lowest-order theory. Figure 5(e) shows that four-phonon scattering rates for the optic phonons are much larger than their three-phonon counterparts at $300 \mathrm{~K}$ for most of the optic phonon bandwidth. Surprisingly, this finding is true even at a low temperature of $100 \mathrm{~K}$, where the influence of four-phonon scattering is expected to be weak even for strongly anharmonic materials [14]. The AOAO and $O O O O$ four-phonon processes dominate the scattering as shown in Supplemental Material, Sec. S9, Figs. S23(c) and S24(d) [34]. For AlSb, optic phonon contributions dominate the calculated RT $\kappa_{\text {Pure }}^{(3)}$, so including four-phonon scattering gives substantially reduced $\kappa_{\text {Pure }}^{(3+4)}$, indicating that the lowest-order theory is failing drastically. In fact, our results indicate that there is very little hope for identifying materials with large contributions to $\kappa$ coming from the optic phonons, since (a) at moderate-to-high temperatures, fourphonon scattering dominates any frequency region of weak three-phonon scattering for the optic phonons driven by the $A A O$ and $A O O \# 2$ selection rules, thereby significantly lowering $\kappa$, while (b) at low temperatures, where 
four-phonon scattering rates can get weaker than their threephonon counterparts, the heat capacity of the optic phonons becomes vanishingly small owing to their high-frequency scale (see Supplemental Material, Sec. S7 [34]).

An interesting additional failure of the lowest-order theory for AlSb is its prediction of a large isotope effect (enhancement in $\kappa$ upon isotopic enrichment), which is seen in the difference between the dashed black and dashed red curves in Fig. 3(b). This effect is quantified by $P=100\left(\kappa_{\text {Pure }} / \kappa_{\text {Nat }}-1\right)$, which remains above $50 \%$ between 100 and $300 \mathrm{~K}$, ignoring four-phonon interactions. In compounds where the heavy-to-light ratio of the masses of the two constituent atoms is large, phonon-isotope scattering is weak throughout the BZ if the vibrating atom is isotopically pure $[32,57]$. This behavior occurs in AlSb $\left(m_{\mathrm{Sb}} / m_{\mathrm{Al}}=4.5\right)$, where the light, isotopically pure $\mathrm{Al}$ atoms dominate the vibrational component of the optic modes while the heavy $\mathrm{Sb}$ atoms, which have a large isotope mix $\left(57 \%{ }^{121} \mathrm{Sb}, 43 \%{ }^{123} \mathrm{Sb}\right)$, hardly move. The resulting weak phonon-isotope scattering shows a striking contrast to the opposite behavior in BAs, where the heavy atom (As) is isotopically pure but the light atom (B) is not [compare phonon-isotope scattering rates in Figs. 5(d) and 5(e)]. Nevertheless, this weak phonon-isotope scattering strongly suppresses $\kappa_{\text {Pure }}^{(3)}$ in AlSb because of the already weak $A O O$ scattering. When four-phonon interactions are included, the $P$ of AlSb drops to between $10 \%$ and $1 \%$ in the same temperature range. Note that the calculated $\kappa_{\mathrm{Nat}}^{(3+4)}$ is in much better agreement with the measured $\kappa$ data in Fig. 3(b) than $\kappa_{\text {Nat }}^{(3)}$.

We note that a large suppression of anharmonic optic phonon lifetimes and of thermal conductivity by fourphonon scattering was predicted in prior work for BAs [13] and AlSb [20]. These works attributed the large lowestorder optic phonon lifetimes to freezing out of $A A O$ scattering by the large $A-O$ gaps. While it is true that $A A O$ scattering is limited, this does not explain why $A O O$ scattering is weak in BAs and AlSb. As discussed above, BSb also has a large $A-O$ gap that removes $A A O$ processes but has much smaller contributions to $\kappa_{\text {Pure }}^{(3)}$ than in either BAs or AlSb, because its larger LO-TO splitting results in larger minimum $A O O$ scattering rates. The new $A O O$ selection rule identified in the present work provides the missing explanation for why $A O O$ scattering becomes weak in both BAs and AlSb but not in BSb.

\section{Other compounds: GaP, AlAs, GaAs, and AlP}

The heavy-to-light atom mass ratios in $\mathrm{GaP}(2.3)$ and AlAs (2.6) are large enough for $A A O$ and $A O O \# 1$ selection rules to create frequency windows where only $A A A$ scattering occurs. Even though no type 2 selection rules are activated in these regions, the lack of $A A O$ and $A O O$ scattering in these frequency windows gives relatively weak three-phonon scattering, and correspondingly large contributions to $\kappa^{(3)}$ result. Four-phonon scattering suppresses $\kappa^{(3)}$ by around $25 \%$ at $300 \mathrm{~K}$ and around $45 \%$ at $750 \mathrm{~K}$.

Relatively small reductions are found in the calculated values of $\kappa^{(3)}$ in AlP and GaAs upon inclusion of fourphonon scattering - around 10\%-15\% at RT. The constituent elements of the two compounds have similar masses resulting in small $A-O$ gaps, so $A A O$ scattering extends throughout the range of the acoustic and the optic phonons [Figs. S8(c) for GaAs and S7(b) for AlP [34] ]. Neither of the $A A A$ selection rules are activated by features in the phonon dispersions for the two materials.

The results for AlP and GaAs highlight the fact that anomalous suppression of $\kappa^{(3)}$ by four-phonon scattering is unlikely to occur for strongly bonded compounds with similar masses, since no frequency window is opened by the type 1 selection rules. A frequency window will also not occur in strongly polar materials having large LO-TO splitting and large optic phonon bandwidths. An example of this behavior is found is $\mathrm{MgO}$ [19], which has no frequency window opened by the selection rules. We calculated the four-phonon scattering rates in $\mathrm{MgO}$ and found them to be comparable to those in the non-BCN zinc blende compounds. Since no selection rules are activated, three-phonon scattering is also strong. A reduction in $\kappa^{(3)}$ of about $15 \%$ is found at RT, consistent with that found in GaAs and AlP.

\section{FOUR-PHONON SCATTERING AND ITS WEAKNESS IN COMPOUNDS WITH BORON, CARBON, OR NITROGEN}

Comparison of the four-phonon scattering rates for the 17 materials studied shows several striking features. First, the four-phonon scattering rates generally increase with increasing frequency, being the strongest for the optic phonons. In particular, they are far larger than the small lowest-order optic phonon scattering rates in BAs and $\mathrm{AlSb}$ responsible for the erroneously large contributions to $\kappa$. As noted above, this finding suggests that it is not likely to be possible to get large contributions from optic phonons in any compound, and any findings to the contrary in the lowest-order phonon-phonon theory are likely incomplete and point simply to the need to consider four-phonon interactions to obtain accurate results. Nevertheless, the dominance of four-phonon anharmonic decay rates for the optic phonons compared with their three-phonon counterparts that we find in BAs, AlSb, InP, AlAs, and BSb around $\Gamma$ [see Figs. 5(d)-5(f), S14(c), and S13(c) [34], respectively] suggests that these signatures should be observable in, e.g., temperature-dependent Raman linewidth measurements.

Second, the four-phonon scattering rates for the nine non-BCN compounds, i.e., those that do not contain B, C, or $\mathrm{N}$ atoms (GaAs, GaP, GaSb, AlSb, AlP, AlAs, InP, InAs, and $\mathrm{InSb}$ ), are remarkably similar in magnitude. An example of this similarity is seen in Figs. 5(e) and 5(f) for AlSb and InP, respectively. Comparisons for the other 
compounds can be made in Figs. 7(d)-7(f) and in Figs. S14(b), S14(c), S15(b) and S15(c) in Supplemental Material [34]. These similarities across materials are in striking contrast to the analogous lowest-order scattering rates. This contrast highlights a fundamental difference between the lowest-order phonon-phonon interactions and those of higher order. The lowest-order processes are constrained by selection rules; features in the phonon dispersions that activate these selection rules can significantly reduce the lowest-order phonon-phonon scattering rates. In contrast, the phase space for four-phonon scattering shows remarkably little structure across materials, being immune to shifts in the $A-O$ gap or to the bunching together of the acoustic or optic branches. The four-phonon scattering rates of the acoustic phonons for all compounds are dominated by $A O A O$ and $A A A A$ processes, and, for those, fourth-order bond anharmonicity and phonon frequency scales act in opposition, maintaining relative uniformity in the scattering strengths. This result is discussed in more detail in Supplemental Material, Sec. S10 [34]. Based on the relatively uniform strength of the four-phonon scattering rates and lack of selection rules influencing them, it seems reasonable to expect that higher-order phononphonon interactions beyond four-phonon scattering (fivephonon and beyond) should play a negligible role in the thermal conductivity of this group of materials.

The most remarkable feature is that the four-phonon scattering rates for the $\mathrm{BCN}$ compounds, i.e., those containing $\mathrm{B}, \mathrm{C}$, or $\mathrm{N}$ atoms (BAs, BSb, BP, BN, SiC, GaN, AlN, and $\mathrm{InN}$ ), are much smaller than those for the nonBCN compounds. This feature is clearly seen by comparing Figs. 5(d)-5(f), which show that the four-phonon scattering rates in BAs are an order of magnitude smaller than those for InP and AlSb. Figures S13-S15 [34] and Fig. 3B in Ref. [18] show that the four-phonon scattering rates for $\mathrm{BSb}, \mathrm{BP}, \mathrm{BN}, \mathrm{SiC}, \mathrm{GaN}$, AlN, and InN are similarly small compared to their non-BCN counterparts. The four-phonon scattering rates at $750 \mathrm{~K}$ show the same trends as those identified at RT. We have traced this behavior to fundamental bonding differences between $\mathrm{BCN}$ and non-BCN compounds. BCN compounds have stronger bonding, reflected in their smaller lattice constants and higher phonon frequency scales compared to the non-BCN compounds. However, the fourth-order anharmonic component of the bonds in the BCN compounds tends to be weaker relative to the harmonic component, compared with those in the non-BCN compounds. The implications of this finding are discussed below. Interestingly, recent calculations of thermal transport in $\mathrm{ZrC}$ [21], a metal with rocksalt structure, also show unusually weak four-phonon scattering rates and correspondingly small reductions in the phononphonon limited $\kappa$, consistent with our findings for the zinc blende BCN compounds.

Exploiting our finding that the $A O A O$ processes are dominant in all the considered materials, we have developed a simple model that captures the weaker fourphonon scattering strength in the $\mathrm{BCN}$ compounds compared with the non-BCN materials. This model is discussed in Supplemental Material, Sec. S10, and findings are plotted in Fig. S27 [34].

\section{DISCUSSION}

From the above findings, we make the following additional observations.

\section{A. Significance of weak four-phonon scattering in BCN compounds}

While the $\kappa$ of BAs is significantly suppressed by fourphonon scattering, it still achieves an ultrahigh RT $\kappa_{\text {Nat }}^{(3+4)}$ value of around $1300 \mathrm{Wm}^{-1} \mathrm{~K}^{-1}$, confirmed by measurements $[17,39,40]$, about $2-3$ times larger than other high- $\kappa$ compounds such as $\mathrm{SiC}, \mathrm{BP}$, copper, and silver. To test the importance of its unusually weak four-phonon processes, we artificially increased the RT four-phonon scattering rates in BAs by a factor of 10, making them comparable to those in the non-BCN compounds. The resulting RT $\kappa$ of BAs is reduced by over $70 \%$ to $330 \mathrm{Wm}^{-1} \mathrm{~K}^{-1}$. Similarly increased four-phonon scattering rates in $\mathrm{GaN}$ and $\mathrm{BP}$ yield $50 \%$ and $30 \%$ decreases, respectively, in the RT $\kappa_{\text {Pure }}$ values for these compounds. This highlights one of the fortuitous benefits that nature gives to the $\mathrm{BCN}$ compounds, without which their $\kappa$ 's could be much lower.

\section{B. High $\kappa$ in non-BCN compounds unlikely}

The larger four-phonon scattering found in the non-BCN materials suggests that finding a material with high $\kappa$ among them will be challenging. Indeed, note that, of the eight compounds above showing small deviations from the predictions of the lowest-order theory upon inclusion of fourphonon scattering, only two (GaAs and AlP) are non-BCN compounds. Conventional guidelines for achieving high $\kappa$, as described by Slack [58], require the combination of light atoms and exceptionally strong bonding, with diamond being the prototype. These criteria already rule out the non-BCN compounds. The alternative paradigm exemplified by BAs [32], in principle, allows for the possibility of a high- $\kappa$ material composed of non-BCN constituent atoms. Such a material would require phonon dispersions for which the selection rules give a small three-phonon scattering phase space in a frequency region where the four-phonon scattering is weak. Such behavior does not occur in any of the studied non-BCN compounds, where, instead, frequency windows with a small total three-phonon phase space coincide with strong four-phonon scattering regions.

\section{Masking of weak $A A A$ and $A O O$ scattering}

The four compounds BAs, BSb, AlSb, and InP showing the catastrophic failures of the lowest-order theory have the 
largest $A-O$ gaps, which remove the $A A O$ processes. This feature is clearly a critical requirement to achieve frequency windows in which anomalously large phonon lifetimes are possible. To highlight the importance of this requirement, consider $\mathrm{BP}$ and $\mathrm{SiC}$ compared with BAs. The $A A A, A O O$, and four-phonon scattering rates in $\mathrm{SiC}, \mathrm{BP}$, and $\mathrm{BAs}$ are similar. Thus, the reason $\mathrm{SiC}$ and $\mathrm{BP}$ have lower $\kappa$ than $\mathrm{BAs}$ stems from the $A A O$ scattering rates, which are much larger than the $A A A$ scattering rates in the frequency range of the $A A A$ minima. To illustrate how important these are, we calculated the $\kappa$ of $\mathrm{SiC}$ and $\mathrm{BP}$ with $A A O$ processes artificially removed. We find that the RT $\kappa_{\text {Pure }}^{(3)}$ of BP in this case is over $6000 \mathrm{Wm}^{-1} \mathrm{~K}^{-1}$, while including fourphonon scattering gives $\kappa_{\text {Pure }}^{(3+4)}$ of around $2600 \mathrm{Wm}^{-1} \mathrm{~K}^{-1}$, roughly twice the corresponding values in BAs. For $\mathrm{SiC}$, the corresponding values are 3800 and $2700 \mathrm{Wm}^{-1} \mathrm{~K}^{-1}$. While it is unfortunately not possible to remove these processes in reality, they could, in principle, be tuned. For example, the application of hydrostatic pressure can preferentially shift the optic phonons to higher frequencies relative to the acoustic phonons. This shift would push $A A O$ processes to higher frequencies exposing the region of weak $A A A$ phase space, which itself increases with pressure $[19,59]$.

Similar behavior is found for the optic phonons. The compounds AlAs, AlP, BP, GaAs, GaSb, GaP, InAs, and InSb have regions of weaker $A O O$ scattering rates due to the $A O O \# 2$ selection rule than does AlSb. Thus, even larger contributions from optic phonons would be predicted from the lowest-order theory for these compounds if not for their comparably small $A-O$ gaps, which give strong $A A O$ scattering where the $A O O$ optic phonon scattering is small. As a result, optic phonons in these compounds contribute negligibly to $\kappa$ even in the lowest-order theory.

\section{Complementary features activating $A A A \# 1$ and $A A A \# 2$ selection rules}

Figure 6 shows that those compounds that more strongly activate $A A A \# 1$ or $A A A \# 2$ selection rules lie on opposite ends of the velocity ratio plot. Thus, compounds that have acoustic branches that are bunched together away from the center of the BZ (BAs, BSb, BP, BN, SiC, AlN, and GaN) and so activate the $A A A \# 1$ selection rule generally have smaller differences between near-zone center LA and TA velocities. In contrast, compounds with large differences in their near-zone center LA and TA velocities (e.g., InSb, InAs, InP, and $\mathrm{GaSb}$ ), and so activate the $A A A \# 2$ selection rule, do not show the acoustic branch bunching behavior or do not have it exposed by the type 1 selection rules.

\section{E. Importance of new type 2 selection rules}

Prior studies have already identified the importance of the $A A A \# 1$ type 2 selection rule in explaining the anomalously weak three-phonon phase space in BAs and $\mathrm{BSb}$. Yet, this selection rule cannot explain the unusually large optic phonon contributions to $\kappa_{\text {Pure }}^{(3)}$ in BAs and AlSb, nor can it explain the large acoustic phonon contributions to $\kappa_{\text {Pure }}^{(3)}$ in InP, InAs, InSb, and GaSb. The two new type 2 selection rules, $A A A \# 2$ and $A O O \# 2$, are required for a full understanding of the behavior in the studied $\mathrm{ZB}$ materials.

\section{F. Restricted spectral contributions to thermal conductivity}

In all of the studied compounds, we find that the dominant contributions to $\kappa$ occur below the highest TA phonon frequency $\nu_{\mathrm{TA}}^{\max }$. Above $\nu_{\mathrm{TA}}^{\max }$, LA phonons exhibit strong decay via $A A A$ processes and also participate in $A A O$ processes, resulting in small contributions to $\kappa$ in this frequency region. The $\nu_{\mathrm{TA}}^{\max }$ are marked in the spectral $\kappa$ contribution figures for each material [Figs. 5(g)-5(i), 7(g)-7(i), and S16-S18 [34] ] to illustrate this behavior.

\section{G. Distinction between phase space and matrix element selection rules}

The selection rules discussed in the present work are connected to the phase space for three-phonon scattering. They depend on specific features being present in phonon dispersions, as described in Sec. II. It is important to distinguish these from a different kind of selection rule, which can cause the matrix elements for phonon-phonon scattering to vanish. Such selection rules have been identified in systems of reduced dimensionality and are connected to the underlying symmetry in some crystals [60-63]. Their incorporation can improve efficiency in schemes for computing phonon-phonon processes, as recently discussed [63].

\section{H. Effect of impurities on the measured $\kappa$}

The large differences seen between the $\kappa$ values calculated only using the lowest-order theory and the measured data for many of the studied materials cannot be explained by possible impurities in the measured samples. The presence of impurities gives a weaker temperature dependence to $\kappa$ compared to the predictions from the lowest-order theory, since phonon-impurity scattering is temperature independent, as described previously [17]. In contrast, the measured $\kappa(T)$ shows a stronger temperature dependence than seen in many of the lowest-order calculations. The calculations including both three-phonon and four-phonon scattering processes also gives stronger $T$ dependence $[13,17]$ and are both qualitatively and quantitatively in agreement with the experimentally measured temperaturedependent $\kappa$.

\section{Impact of selection rules in other materials}

The selection rules on three-phonon scattering are completely general and are not restricted to ZB compounds. 
The creation of frequency windows in Fig. 2 benefits from materials that have low ionicity (e.g., several ZB compounds in this study), which gives relatively small optic phonon bandwidths, and large heavy-to-light mass ratios. For materials with more than two atoms in the unit cell, the additional scattering channels resulting from the increased number of optic phonon branches make the needed frequency windows harder to achieve for acoustic phonons, so the selection rules are less likely to affect the acoustic phonons in such compounds. But large frequency gaps between bands of optic phonons can be found in many compounds [64], so erroneously large anharmonic phonon lifetimes and contributions to $\kappa$ from some optic phonons could occur from activation of selection rules such as $A O O \# 2$ and $O O O$. For such compounds, the selection rule picture can provide useful guidance for understanding measurements giving lower $\kappa$ and stronger temperature dependence of anharmonic phonon decay than predicted from calculations including only three-phonon scattering. In crystals with one atom in the unit cell, only $A A A$ processes occur. We are not aware of any such materials that are semiconductors or insulators, and, in metals, $\kappa$ is typically dominated by the electronic contribution. For the lattice contribution to $\kappa$ in metals, inclusion of electronphonon interactions could mask any influence of the selection rules on the lowest-order phonon-phonon interactions.

\section{SUMMARY}

$A b$ initio theories of the anharmonic properties of materials continue to rely on descriptions that include only the lowest-order phonon-phonon interactions. The vastly different results obtained here for phonon lifetimes and thermal conductivity with and without four-phonon interactions for the many considered materials contradict the prevailing understanding of anharmonic phonon decay and thermal conduction in weakly anharmonic crystals, where four-phonon interactions have frequently been assumed to be unimportant.

We have identified new selection rules on three-phonon scattering dictated entirely by features in the phonon dispersions and have shown that the full set of selection rules listed in Sec. II and the trends found in four-phonon scattering strengths provide a useful framework to understand the anharmonic phonon decay and thermal conductivity of weakly anharmonic insulating crystals. In particular, the new selection rules are critical in explaining the failure of the lowest-order theory in describing thermal transport in many zinc blende compounds.

In compounds that do not contain boron, carbon, or nitrogen, four-phonon scattering suppresses the erroneously large phonon lifetimes and thermal conductivities predicted by the lowest-order theory. Conversely, in compounds that do contain boron, carbon, and nitrogen atoms, large phonon lifetimes and thermal conductivities predicted in the lowest-order theory are less affected because of the exceptionally weak four-phonon interactions. These findings help explain the high thermal conductivities found in technologically important compounds such as BAs, BP, and $\mathrm{SiC}$ and provide critical guidance in the search for new materials with high thermal conductivity.

Inclusion of four-phonon interactions in ab initio anharmonic decay rates and transport calculations is computationally challenging, and such calculations are currently performed by only a small number of groups [12-21]. Recent work has shown that, for strongly anharmonic materials, it is essential to include four-phonon processes [14-16]. The present study shows that four-phonon scattering is much stronger in weakly anharmonic materials that do not containing $\mathrm{B}, \mathrm{C}$, or $\mathrm{N}$ atoms compared with those that do. Consequently, future thermal transport studies of such strongly-bonded compounds may need to include four-phonon scattering in order to obtain accurate results. While this suggestion has been made before [13], the large number of compounds studied in the present work and comparably larger four-phonon scattering strengths found in the non-BCN compounds adds strong quantitative and statistical support to this proposition. Further calculations will be required to fully establish this point.

\section{METHODS}

For all materials, temperature-dependent lattice parameters are obtained by minimizing the Helmholtz free energy $F=\Phi_{0}+F_{H}+F_{A}$, where $\Phi_{0}$ is the energy of the lattice atoms in their equilibrium positions, $F_{H}$ is the harmonic part, and $F_{A}$ is the anharmonic part, which includes both third- and fourth-order terms [14]. To obtain phonon modes, the second-order interatomic force constants (IFCs) are calculated using density functional perturbation theory (DFPT) as implemented in the QUANTUM ESPRESSO package [65]. All calculations in this work are performed using norm-conserving pseudopotentials with the local density approximation exchange correlation functional. Converged parameters for the density functional theory calculations are provided in Supplemental Material, Sec. S14 [34].

The third- and fourth-order anharmonic IFCs required to obtain three-phonon and four-phonon scattering rates are calculated using a thermal stochastic snapshot technique. The method is described in detail in Ref. [14]. Temperaturedependent anharmonic renormalization of phonon modes [14] is included but does not significantly affect phonon modes, scattering rates, and thermal conductivities for the considered materials and temperature ranges. To calculate the thermal conductivity of a material, the Boltzmann equation for phonon transport including three-phonon, four-phonon, and phonon-isotope scattering is solved using an iterative approach for the nonequilibrium phonon distribution function created from an assumed small temperature gradient across the considered materials. For all materials and all temperatures, the phonon Boltzmann 
equation is solved on a $51 \times 51 \times 51 \mathbf{q}$ grid. Four-phonon scattering rates are obtained on $17 \times 17 \times 17 \mathbf{q}$ grids and interpolated to the finer $51 \times 51 \times 51$ q grids (see Supplemental Material, Sec. S13 [34], for details). Full calculations (without interpolation) are also performed on $21 \times 21 \times 21$ q grids to check the convergence of the fourphonon scattering rates (see Supplemental Material, Sec. S12 [34]). The phonon Boltzmann equation and the expressions for the scattering rates are given in the Appendix.

\section{ACKNOWLEDGMENTS}

This work was supported by the Office of Naval Research under a Multidisciplinary University Research Initiative, Grant No. N00014-16-1-2436.

The authors declare no competing financial interests.

N.K.R. and D.B. originated the research. N.K.R. performed the $a b$ initio calculations. N. K. R. and D. B. analyzed the results and wrote the manuscript. Both authors studied, commented on, and edited the manuscript.

\section{APPENDIX: PHONON BOLTZMANN EQUATION, SCATTERING RATES, AND THERMAL CONDUCTIVITY}

The phonon Boltzmann equation for an applied temperature gradient $\Delta T$ is

$$
\mathbf{v}_{\lambda} \cdot \nabla T \frac{\partial n_{\lambda}^{0}}{\partial T}=\left.\frac{\partial n_{\lambda}}{\partial t}\right|_{\text {collisions }},
$$

where $\mathbf{v}_{\lambda}$ is the phonon group velocity and $n_{\lambda}^{0}=$ $1 /\left(e^{\hbar \omega_{\lambda} /\left(k_{B} T\right)}-1\right)$ is the equilibrium Bose distribution at temperature $T$ and for phonon mode $\lambda=(\mathbf{q}, j)$ and frequency $\omega_{\lambda}$.

The nonequilibrium phonon distribution function linearized in assumed small $\Delta T$ is

$$
n_{\lambda}=n_{\lambda}^{0}+n_{\lambda}^{0}\left(n_{\lambda}^{0}+1\right) \mathbf{F}_{\lambda} \cdot(-\nabla T) .
$$

The function $\mathbf{F}_{\lambda}$ is obtained by solving the linearized phonon Boltzmann equation including three-phonon, four-phonon, and phonon-isotope scattering:

$$
\begin{aligned}
\mathbf{F}_{\lambda}= & \mathbf{F}_{\lambda}^{0}+\tau_{\lambda}^{(\text {tot })}\left\{\sum_{\lambda_{1} \lambda_{2}}\left[W_{\lambda \lambda_{1} \lambda_{2}}^{(+)}\left(\mathbf{F}_{\lambda_{2}}-\mathbf{F}_{\lambda_{1}}\right)+\frac{1}{2} W_{\lambda \lambda_{1} \lambda_{2}}^{(-)}\left(\mathbf{F}_{\lambda_{2}}+\mathbf{F}_{\lambda_{1}}\right)\right]+\sum_{\lambda_{1}} W_{\lambda \lambda_{1}}^{\text {iso }} \mathbf{F}_{\lambda_{1}}\right. \\
& \left.+\sum_{\lambda_{1} \lambda_{2} \lambda_{3}}\left[\frac{1}{6} Y_{\lambda \lambda_{1} \lambda_{2} \lambda_{3}}^{(1)}\left(\mathbf{F}_{\lambda_{1}}+\mathbf{F}_{\lambda_{2}}+\mathbf{F}_{\lambda_{3}}\right)+\frac{1}{2} Y_{\lambda \lambda_{1} \lambda_{2} \lambda_{3}}^{(2)}\left(\mathbf{F}_{\lambda_{2}}+\mathbf{F}_{\lambda_{3}}-\mathbf{F}_{\lambda_{1}}\right)+\frac{1}{2} Y_{\lambda \lambda_{1} \lambda_{2} \lambda_{3}}^{(3)}\left(\mathbf{F}_{\lambda_{3}}-\mathbf{F}_{\lambda_{2}}-\mathbf{F}_{\lambda_{1}}\right)\right]\right\},
\end{aligned}
$$

where

$$
\begin{gathered}
\mathbf{F}_{\lambda}^{0}=\hbar \omega_{\lambda} \mathbf{v}_{\lambda} \tau_{\lambda}^{(\mathrm{tot})} / k_{B} T \\
1 / \tau_{\lambda}^{(\mathrm{tot})}=1 / \tau_{\lambda}^{(3-\mathrm{ph})}+1 / \tau_{\lambda}^{(4-\mathrm{ph})}+1 / \tau_{\lambda}^{(\mathrm{ph}-\mathrm{iso})}, \\
1 / \tau_{\lambda}^{(3-\mathrm{ph})}=\sum_{\lambda_{1} \lambda_{2}}\left[W_{\lambda \lambda_{1} \lambda_{2}}^{(+)}+\frac{1}{2} W_{\lambda \lambda_{1} \lambda_{2}}^{(-)}\right] \\
1 / \tau_{\lambda}^{(\mathrm{ph}-\mathrm{iso})}=\sum_{\lambda_{1}} W_{\lambda \lambda_{1}}^{\mathrm{iso}}, \\
1 / \tau_{\lambda}^{(4-\mathrm{ph})}=\sum_{\lambda_{1} \lambda_{2} \lambda_{3}}\left[\frac{1}{6} Y_{\lambda \lambda_{1} \lambda_{2} \lambda_{3}}^{(1)}+\frac{1}{2} Y_{\lambda \lambda_{1} \lambda_{2} \lambda_{3}}^{(2)}+\frac{1}{2} Y_{\lambda \lambda_{1} \lambda_{2} \lambda_{3}}^{(3)}\right]
\end{gathered}
$$

The three-phonon scattering probabilities are

$$
\begin{aligned}
& W_{\lambda \lambda_{1} \lambda_{2}}^{(+)}=\frac{2 \pi}{\hbar^{2}}\left|\Phi_{\lambda \lambda_{1}\left(-\lambda_{2}\right)}\right|^{2}\left(n_{\lambda_{1}}^{0}-n_{\lambda_{2}}^{0}\right) \delta\left(\omega_{\lambda}+\omega_{\lambda_{1}}-\omega_{\lambda_{2}}\right), \\
& W_{\lambda \lambda_{1} \lambda_{2}}^{(-)}=\frac{2 \pi}{\hbar^{2}}\left|\Phi_{\lambda\left(-\lambda_{1}\right)\left(-\lambda_{2}\right)}\right|^{2}\left(1+n_{\lambda_{1}}^{0}+n_{\lambda_{2}}^{0}\right) \delta\left(\omega_{\lambda}-\omega_{\lambda_{1}}-\omega_{\lambda_{2}}\right) .
\end{aligned}
$$

The four-phonon scattering probabilities are 


$$
\begin{aligned}
& Y_{\lambda \lambda_{1} \lambda_{2} \lambda_{3}}^{(1)}=\frac{2 \pi}{\hbar^{2}}\left|\Phi_{\lambda\left(-\lambda_{1}\right)\left(-\lambda_{2}\right)\left(-\lambda_{3}\right)}\right|^{2} \frac{n_{\lambda_{1}}^{0} n_{\lambda_{2}}^{0} n_{\lambda_{3}}^{0}}{n_{\lambda}^{0}} \delta\left(\omega_{\lambda}-\omega_{\lambda_{1}}-\omega_{\lambda_{2}}-\omega_{\lambda_{3}}\right), \\
& Y_{\lambda \lambda_{1} \lambda_{2} \lambda_{3}}^{(2)}=\frac{2 \pi}{\hbar^{2}}\left|\Phi_{\lambda \lambda_{1}\left(-\lambda_{2}\right)\left(-\lambda_{3}\right)}\right|^{2} \frac{\left(1+n_{\lambda_{1}}^{0}\right) n_{\lambda_{2}}^{0} n_{\lambda_{3}}^{0}}{n_{\lambda}^{0}} \delta\left(\omega_{\lambda}+\omega_{\lambda_{1}}-\omega_{\lambda_{2}}-\omega_{\lambda_{3}}\right), \\
& Y_{\lambda \lambda_{1} \lambda_{2} \lambda_{3}}^{(3)}=\frac{2 \pi}{\hbar^{2}}\left|\Phi_{\lambda \lambda_{1} \lambda_{2}\left(-\lambda_{3}\right)}\right|^{2} \frac{\left(1+n_{\lambda_{1}}^{0}\right)\left(1+n_{\lambda_{2}}^{0}\right) n_{\lambda_{3}}^{0}}{n_{\lambda}^{0}} \delta\left(\omega_{\lambda}+\omega_{\lambda_{1}}+\omega_{\lambda_{2}}-\omega_{\lambda_{3}}\right) .
\end{aligned}
$$

The three-phonon and four-phonon matrix elements are

$$
\begin{aligned}
\Phi_{\lambda \lambda_{1} \lambda_{2}}= & \Phi_{\mathbf{q} s, \mathbf{q}_{1} s_{1}, \mathbf{q}_{2} s_{2}} \\
= & (\hbar / 2)^{3 / 2}\left(1 / N_{0}^{1 / 2}\right)\left[\omega_{\mathbf{q} s} \omega_{\mathbf{q}_{1} s_{1}} \omega_{\mathbf{q}_{2} s_{2}}\right]^{-1 / 2} \sum_{N P} \sum_{\mu \nu \pi} \sum_{\alpha \beta \gamma} \Phi_{\alpha \beta \gamma}(0 \mu, N \nu, P \pi)\left(M_{\mu} M_{\nu} M_{\pi}\right)^{-1 / 2} \\
& \times e^{i \mathbf{q}_{1} \cdot \mathbf{R}(N)} e^{i \mathbf{q}_{2} \cdot \mathbf{R}(P)} w_{\alpha}(\mathbf{q} s, \mu) w_{\beta}\left(\mathbf{q}_{1} s_{1}, \nu\right) w_{\gamma}\left(\mathbf{q}_{2} s_{2}, \pi\right)
\end{aligned}
$$

and

$$
\begin{aligned}
\Phi_{\lambda \lambda_{1} \lambda_{2} \lambda_{3}}= & \Phi_{\mathbf{q} s, \mathbf{q}_{1} s_{1}, \mathbf{q}_{2} s_{2}, \mathbf{q}_{3} s_{3}} \\
= & (\hbar / 2)^{2}\left(1 / N_{0}\right)\left[\omega_{\mathbf{q} s} \omega_{\mathbf{q}_{1} s_{1}} \omega_{\mathbf{q}_{2} s_{2}} \omega_{\mathbf{q}_{3} s_{3}}\right]^{-1 / 2} \sum_{N P Q} \sum_{\mu \nu \pi \rho} \sum_{\alpha \beta \gamma \eta} \Phi_{\alpha \beta \gamma \eta}(0 \mu, N \nu, P \pi, Q \rho)\left(M_{\mu} M_{\nu} M_{\pi} M_{\rho}\right)^{-1 / 2} \\
& \times e^{i \mathbf{q}_{1} \cdot \mathbf{R}(N)} e^{i \mathbf{q}_{2} \cdot \mathbf{R}(P)} e^{i \mathbf{q}_{3} \cdot \mathbf{R}(Q)} w_{\alpha}(\mathbf{q} s, \mu) w_{\beta}\left(\mathbf{q}_{1} s_{1}, \nu\right) w_{\gamma}\left(\mathbf{q}_{2} s_{2}, \pi\right) w_{\eta}\left(\mathbf{q}_{3} s_{3}, \rho\right),
\end{aligned}
$$

respectively, where $w_{\alpha}(\mathbf{q} s, \mu)$ is the $\alpha$ th component of the eigenvector $\mathbf{w}(\lambda, \mu)=\mathbf{w}(\mathbf{q} s, \mu)$ for a phonon with wave vector $\mathbf{q}$ and polarization $s$ and for the basis atom $\mu$ and $N_{0}$ is the number of atoms in the supercell (or, equivalently, the number of $\mathbf{q}$ points in the commensurate first BZ). The phonon-isotope scattering probabilities are

$W_{\lambda \lambda_{1}}^{\text {iso }}=\frac{\pi \omega_{\lambda}^{2}}{2 N_{0}} \sum_{b} g_{2}(b)\left|\mathbf{w}(\lambda, b) \cdot \mathbf{w}^{*}\left(\lambda_{1}, b\right)\right|^{2} \delta\left(\omega_{\lambda}-\omega_{\lambda_{1}}\right)$,

where $g_{2}(b)=\left(1 / \bar{M}_{b}^{2}\right) \sum_{a} f_{a b}\left(M_{a b}-\bar{M}_{b}\right)^{2}$ is the mass variance parameter with $f_{a b}$ and $M_{a b}$ being the concentration and mass of the $a$ th isotope of the $b$ th atom, respectively, and $\bar{M}_{b}$ is the average mass of the $b$ th atom. We find that the momentum-relaxing umklapp processes are comparable to or stronger than the momentumconserving normal processes for the four-phonon interactions for all 17 materials and at all temperatures considered in this work (see Supplemental Material, Sec. S11 [34]). As a result, treating the four-phonon scattering within a relaxation time approximation (RTA), i.e., removing the last bracketed term in Eq. (A3), gives a negligible difference compared to the full solution of Eq. (A3). We have confirmed that (a) solving the full phonon Boltzmann equation, which explicitly differentiates normal and umklapp three-phonon and four-phonon interactions, and (b) solving the partial phonon Boltzmann equation with four-phonon scattering under the RTA by removing the last bracketed term in Eq. (A3) results in a negligible difference in $\kappa^{(3+4)}$ at $300 \mathrm{~K}$ for all 17 materials in this study. Therefore, the calculations at all other temperatures presented in this work are obtained by solving the phonon Boltzmann equation with the four-phonon scattering terms treated under the RTA, to reduce the computational cost of these temperature-dependent calculations for the 17 materials.

After solving the phonon Boltzmann equation for $\mathbf{F}_{\lambda}$, the phonon thermal conductivity is calculated as

$$
\kappa_{\alpha \beta}=\sum_{\lambda} C_{\lambda} v_{\lambda \alpha} F_{\lambda \beta},
$$

where $C_{\lambda}=(1 / V) k_{B}\left(\partial n_{\lambda}^{0} / \partial T\right)$ is the mode-specific heat.

[1] R. Peierls, Zur Kinetischen Theorie der Wärmeleitung in Kristallen, Ann. Phys. (Berlin) 395, 1055 (1929).

[2] A. A. Maradudin and A. E. Fein, Scattering of Neutrons by an Anharmonic Crystal, Phys. Rev. 128, 2589 (1962).

[3] R. A. Cowley, Anharmonic Crystals, Rep. Prog. Phys. 31, 123 (1968).

[4] T. H. Geballe and G. W. Hull, Seebeck Effect in Germanium, Phys. Rev. 94, 1134 (1954).

[5] J. Fu, Q. Xu, G. Han, B. Wu, C. H. A. Huan, M. L. Leek, and T. C. Sum, Hot Carrier Cooling Mechanisms in Halide Perovskites, Nat. Commun. 8, 1 (2017). 
[6] J. Yang, X. Wen, H. Xia, R. Sheng, Q. Ma, J. Kim, P. Tapping, T. Harada, T. W. Kee, F. Huang, Y.-B. Cheng, M. Green, A. Ho-Baillie, S. Huang, S. Shrestha, R. Patterson, and G. Conibeer, Acoustic-Optical Phonon Up-Conversion and Hot-Phonon Bottleneck in Lead-Halide Perovskites, Nat. Commun. 8, 1 (2017).

[7] D. W. Pohl and V. Irniger, Observation of Second Sound in NaF by Means of Light Scattering, Phys. Rev. Lett. 36, 480 (1976).

[8] S. Huberman, R. A. Duncan, K. Chen, B. Song, V. Chiloyan, Z. Ding, A. A. Maznev, G. Chen, and K. A. Nelson, Observation of Second Sound in Graphite at Temperatures above $100 \mathrm{~K}$, Science 364, 375 (2019).

[9] L. Lindsay, C. Hua, X. L. Ruan, and S. Lee, Survey of Ab Initio Phonon Thermal Transport, Mater. Today Phys. 7, 106 (2018).

[10] A. J. H. McGaughey, A. Jain, H.-Y. Kim, and B. Fu, Phonon Properties and Thermal Conductivity from First Principles, Lattice Dynamics, and the Boltzmann Transport Equation, J. Appl. Phys. 125, 011101 (2019).

[11] L. Lindsay, A. Katre, A. Cepellotti, and N. Mingo, Perspective on Ab Initio Phonon Thermal Transport, J. Appl. Phys. 126, 050902 (2019).

[12] T. Feng and X. Ruan, Quantum Mechanical Prediction of Four-Phonon Scattering Rates and Reduced Thermal Conductivity of Solids, Phys. Rev. B 93, 045202 (2016).

[13] T. Feng, L. Lindsay, and X. Ruan, Four-Phonon Scattering Significantly Reduces Intrinsic Thermal Conductivity of Solids, Phys. Rev. B 96, 161201 (2017).

[14] N. K. Ravichandran and D. Broido, Unified First-Principles Theory of Thermal Properties of Insulators, Phys. Rev. B 98, 085205 (2018).

[15] Y. Xia, Revisiting Lattice Thermal Transport in PbTe: The Crucial Role of Quartic Anharmonicity, Appl. Phys. Lett. 113, 073901 (2018).

[16] Y. Xia and M. K. Y. Chan, Anharmonic Stabilization and Lattice Heat Transport in Rocksalt $\beta$-GeTe, Appl. Phys. Lett. 113, 193902 (2018).

[17] F. Tian et al., Unusual High Thermal Conductivity in Boron Arsenide Bulk Crystals, Science 361, 582 (2018).

[18] K. Chen et al., Ultrahigh Thermal Conductivity in IsotopeEnriched Cubic Boron Nitride, Science 367, 555 (2020).

[19] N. K. Ravichandran and D. Broido, Non-monotonic Pressure Dependence of the Thermal Conductivity of Boron Arsenide, Nat. Commun. 10, 1 (2019).

[20] X. Yang, T. Feng, J. Li, and X. Ruan, Stronger Role of Four-Phonon Scattering than Three-Phonon Scattering in Thermal Conductivity of III-V Semiconductors at Room Temperature, Phys. Rev. B 100, 245203 (2019).

[21] T. A. Mellan, A. Aziz, Y. Xia, R. Grau-Crespo, and A. I. Duff, Electron and Phonon Interactions and Transport in the Ultrahigh-Temperature Ceramic ZrC, Phys. Rev. B 99, 094310 (2019).

[22] O. Hellman, I. A. Abrikosov, and S. I. Simak, Lattice Dynamics of Anharmonic Solids from First Principles, Phys. Rev. B 84, 180301 (2011).

[23] O. Hellman, P. Steneteg, I. A. Abrikosov, and S. I. Simak, Temperature Dependent Effective Potential Method for Accurate Free Energy Calculations of Solids, Phys. Rev. B 87, 104111 (2013).
[24] I. Errea, M. Calandra, and F. Mauri, First-Principles Theory of Anharmonicity and the Inverse Isotope Effect in Superconducting Palladium-Hydride Compounds, Phys. Rev. Lett. 111, 177002 (2013).

[25] P. Souvatzis, O. Eriksson, M. I. Katsnelson, and S. P. Rudin, Entropy Driven Stabilization of Energetically Unstable Crystal Structures Explained from First Principles Theory, Phys. Rev. Lett. 100, 095901 (2008).

[26] A. van Roekeghem, J. Carrete, and N. Mingo, Anomalous Thermal Conductivity and Suppression of Negative Thermal Expansion in $\mathrm{ScF}_{3}$, Phys. Rev. B 94, 020303 (2016).

[27] G. A. S. Ribeiro, L. Paulatto, R. Bianco, I. Errea, F. Mauri, and M. Calandra, Strong Anharmonicity in the Phonon Spectra of PbTe and SnTe from First Principles, Phys. Rev. B 97, 014306 (2018).

[28] T. Tadano and S. Tsuneyuki, Self-Consistent Phonon Calculations of Lattice Dynamical Properties in Cubic $\mathrm{SrTiO}_{3}$ with First-Principles Anharmonic Force Constants, Phys. Rev. B 92, 054301 (2015).

[29] T. Tadano and S. Tsuneyuki, First-Principles Lattice Dynamics Method for Strongly Anharmonic Crystals, J. Phys. Soc. Jpn. 87, 041015 (2018).

[30] P. B. Allen, Anharmonic Phonon Quasiparticle Theory of Zero-Point and Thermal Shifts in Insulators: Heat Capacity, Bulk Modulus, and Thermal Expansion, Phys. Rev. B 92, 064106 (2015).

[31] M. Lax, P. Hu, and V. Narayanamurti, Spontaneous Phonon Decay Selection Rule: $n$ and $u$ Processes, Phys. Rev. B 23, 3095 (1981).

[32] L. Lindsay, D. A. Broido, and T. L. Reinecke, FirstPrinciples Determination of Ultrahigh Thermal Conductivity of Boron Arsenide: A Competitor for Diamond?, Phys. Rev. Lett. 111, 025901 (2013).

[33] S. Mukhopadhyay, L. Lindsay, and D. S. Parker, Optic Phonon Bandwidth and Lattice Thermal Conductivity: The Case of $\mathrm{Li}_{2} \mathrm{X}(X=\mathrm{O}, \mathrm{S}, \mathrm{Se}, \mathrm{Te})$, Phys. Rev. B 93, 224301 (2016).

[34] See Supplemental Material at http://link.aps.org/ supplemental/10.1103/PhysRevX.10.021063 for several sections of additional information including details of convergence of the first-principles calculations, phonon dispersions and other phonon properties of a few materials not exhaustively covered in the main text.

[35] J. M. Ziman, Electrons and Phonons (Oxford University, New York, 1960).

[36] L. Lindsay and D. A. Broido, Three-Phonon Phase Space and Lattice Thermal Conductivity in Semiconductors, J. Phys. Condens. Matter 20, 165209 (2008).

[37] W. Li, J. Carrete, N. A. Katcho, and N. Mingo, ShengBTE: A Solver of the Boltzmann Transport Equation for Phonons, Comput. Phys. Commun. 185, 1747 (2014).

[38] S. Lee, K. Esfarjani, T. Luo, J. Zhou, Z. Tian, and G. Chen, Resonant Bonding Leads to Low Lattice Thermal Conductivity, Nat. Commun. 5, 3525 (2014).

[39] J. S. Kang, M. Li, H. Wu, H. Nguyen, and Y. Hu, Experimental Observation of High Thermal Conductivity in Boron Arsenide, Science 361, 575 (2018).

[40] S. Li, Q. Zheng, Y. Lv, X. Liu, X. Wang, P. Y. Huang, D. G. Cahill, and B. Lv, High Thermal Conductivity in Cubic Boron Arsenide Crystals, Science 361, 579 (2018). 
[41] V. M. Muzhdaba, A. Y. Nashelsky, P. V. Tamarin, and S. S. Shalyt, Thermal Conductivity and Thermo-EMF of AlSb and Gap at Low Temperatures, Sov. Phys. Solid State 10, 2265 (1969).

[42] E. F. Steigmeier and I. Kudman, Acoustical-Optical Phonon Scattering in Ge, Si, and III-V Compounds, Phys. Rev. 141, 767 (1966).

[43] S. A. Aliev, A. I. A. Nashelskii, and S. S. Shalyt, Thermal Conductivity and Thermoelectric Power of n-Type Indium Phosphide at Low Temperatures (Temperature Dependence of Heat Conductivity and Thermal EMF in n-Type InP), Sov. Phys. Solid State 7, 1287 (1965).

[44] I. Kudman and E. F. Steigmeier, Thermal Conductivity and Seebeck Coefficient of InP, Phys. Rev. 133, A1665 (1964).

[45] M. G. Holland, Phonon Scattering in Semiconductors from Thermal Conductivity Studies, Phys. Rev. 134, A471 (1964).

[46] D. G. Arasly, R. N. Ragimov, and M. I. Aliev, Characteristics of Phonon Scattering in $\mathrm{Ga}_{x} \mathrm{In}_{1-x}$ as Solid Solutions, Sov. Phys. Semicond. 24, 225 (1990).

[47] R. Bowers, J. E. Bauerle, and A. J. Cornish, $\operatorname{In} A s_{1-x} P_{x}$ as a Thermoelectric Material, J. Appl. Phys. 30, 1050 (1959).

[48] G. Le Guillou and H. J. Albany, Phonon Conductivity of InAs, Phys. Rev. B 5, 2301 (1972).

[49] P. V. Tamarin and S. S. Shalyt, Thermal Conductivity and Thermoelectric Power of Indium Arsenide at Low Temperatures, Sov. Phys. Semicond. 5, 1097 (1971).

[50] S. S. Shalyt, P. V. Tamarin, and V. S. Ivleva, The Scattering of Long Wavelength Phonons by Current Carriers in InSb, Phys. Lett. A 32, 29 (1970).

[51] A. Katre, J. Carrete, B. Dongre, G. K. H. Madsen, and N. Mingo, Exceptionally Strong Phonon Scattering by B Substitution in Cubic SiC, Phys. Rev. Lett. 119, 075902 (2017).

[52] J. S. Kang, H. Wu, and Y. Hu, Thermal Properties and Phonon Spectral Characterization of Synthetic Boron Phosphide for High Thermal Conductivity Applications, Nano Lett. 17, 7507 (2017).

[53] Q. Zheng, S. Li, C. Li, Y. Lv, X. Liu, P. Y. Huang, D. A. Broido, B. Lv, and D. G. Cahill, High Thermal Conductivity in Isotopically Enriched Cubic Boron Phosphide, Adv. Funct. Mater. 28, 1805116 (2018).

[54] D. Morelli, J. Heremans, C. Beetz, W. S. Woo, G. L. Harris, and C. Taylor, Carrier Concentration Dependence of the Thermal Conductivity of Silicon Carbide, Inst. Phys. Conf. Ser. N 137, 313 (1993).

[55] L. Lindsay, D. A. Broido, and T. L. Reinecke, Ab Initio Thermal Transport in Compound Semiconductors, Phys. Rev. B 87, 165201 (2013).

[56] R. M. Wentzcovitch, M. L. Cohen, and P. K. Lam, Theoretical Study of BN, BP, and BAs at High Pressures, Phys. Rev. B 36, 6058 (1987).

[57] L. Lindsay, D. A. Broido, and T. L. Reinecke, PhononIsotope Scattering and Thermal Conductivity in Materials with a Large Isotope Effect: A First-Principles Study, Phys. Rev. B 88, 144306 (2013).

[58] G. A. Slack, Nonmetallic Crystals with High Thermal Conductivity, J. Phys. Chem. Solids 34, 321 (1973).

[59] L. Lindsay, D. A. Broido, J. Carrete, N. Mingo, and T. L. Reinecke, Anomalous Pressure Dependence of Thermal Conductivities of Large Mass Ratio Compounds, Phys. Rev. B 91, 121202(R) (2015).

[60] L. Lindsay, D. A. Broido, and N. Mingo, Lattice Thermal Conductivity of Single-Walled Carbon Nanotubes: Beyond the Relaxation Time Approximation and Phonon-Phonon Scattering Selection Rules, Phys. Rev. B 80, 125407 (2009).

[61] L. Lindsay, D. A. Broido, and N. Mingo, Flexural Phonons and Thermal Transport in Graphene, Phys. Rev. B 82, 115427 (2010).

[62] T. Pandey, C. A. Polanco, V. R. Cooper, D. S. Parker, and L. Lindsay, Symmetry-Driven Phonon Chirality and Transport in One-Dimensional and Bulk $\mathrm{Ba}_{3} \mathrm{~N}$-Derived Materials, Phys. Rev. B 98, 241405 (2018).

[63] A. Cammarata, Phonon-Phonon Scattering Selection Rules and Control: An Application to Nanofriction and Thermal Transport, RSC Adv. 9, 37491 (2019).

[64] http://phonondb.mtl.kyoto-u.ac.jp/.

[65] P. Giannozzi et al., QUANTUM ESPRESSO: A Modular and Open-Source Software Project for Quantum Simulations of Materials, J. Phys. Condens. Matter 21, 395502 (2009). 